\title{
The Sen limit
}

\author{
Adrian Clingher, Ron Donagi and Martijn Wijnholt
}

\begin{abstract}
$F$-theory compactifications on elliptic Calabi-Yau manifolds may be related to IIb compactifications by taking a certain limit in complex structure moduli space, introduced by A. Sen. The limit has been characterized on the basis of $S L(2, \mathbf{Z})$ monodromies of the elliptic fibration. Instead, we introduce a stable version of the Sen limit. In this picture the elliptic Calabi-Yau splits into two pieces, a $\mathbf{P}^{1}$-bundle and a conic bundle, and the intersection yields the IIb space-time. We get a precise match between $F$-theory and perturbative type IIb. The correspondence is holographic, in the sense that physical quantities seemingly spread in the bulk of the $F$-theory Calabi-Yau may be rewritten as expressions on the log boundary. Smoothing the $F$-theory Calabi-Yau corresponds to summing up the $D(-1)$-instanton corrections to the IIb theory.
\end{abstract}

1 Introduction

2 The IIb limit as a stable degeneration

2.1 Sen's description of the limit

2.2 Stable version of the Sen degeneration

2.3 Rational surfaces and $G$-bundles on an elliptic curve 622

2.4 The cylinder map for conic bundles

2.5 Asymptotics of the superpotential in the Sen limit

3 Further aspects of the correspondence

3.1 Tadpoles, Euler character and Chern-Schwartz-Macpherson classes

3.2 Singularities of $S O(2 n)$ spectral covers 


\section{Introduction}

$F$-theory was introduced to study vacua with 7 -branes and varying axiodilaton [1]. Its most common definition (which however covers only one branch of the moduli space [2]) is obtained by considering a weakly coupled $M$-theory compactification on an elliptically fibered Calabi-Yau $\pi: Y \rightarrow B$, and taking the limit as the area of the elliptic fibers shrinks to zero. The axio-dilaton of type IIb supergravity on $B$ is identified with the modular parameter of the elliptic fiber. The main advantage of this point of view is that while the axio-dilaton is a complicated multi-valued function over $B$, the geometric description $\pi: Y \rightarrow B$ allows for a global description without branch cuts.

Since $F$-theory was originally thought of as a strong coupling generalization of type IIb, it was natural to look for a precise limit of the elliptically fibered Calabi-Yau in which one should recover the perturbative IIb theory. Such a limit was proposed by A. Sen, and is now commonly referred to as the Sen limit of an F-theory compactification. In a nutshell, Sen's idea is the following. Since the elliptic Calabi-Yau has a section, it can be put in Weierstrass form

$$
y^{2}=x^{3}+f x+g
$$

The modular parameter $\tau$ is identified with the varying axio-dilaton $i e^{-\phi}+a$ of type IIb. The $j$-function of the elliptic fiber can now be expressed as

$$
j(\tau)=\frac{4(24 f)^{3}}{\Delta}, \quad \Delta=4 f^{3}+27 g^{2}
$$

To recover perturbative IIb, we want $g_{s} \rightarrow 0$, so we want $\tau \rightarrow i \infty$ except possibly at the location of the 7 -branes. The most generic way to do this is 
as follows. We express the Weierstrass coefficients as

$$
f \sim b_{2}^{2}+\mathcal{O}(\epsilon), \quad g \sim b_{2}^{3}+\mathcal{O}(\epsilon)
$$

By picking suitable coefficients, the leading terms cancel and we get $\Delta \propto \epsilon$. Then as $\epsilon \rightarrow 0$ we have $\tau \rightarrow i \infty$ (and hence $g_{s} \rightarrow 0$ ) except at $b_{2}=0$. The locus $b_{2}=0$ on $B$ is eventually identified with the orientifold locus in type IIb. The D7-brane locus depends on the $\mathcal{O}(\epsilon)$ terms.

Although this perspective has led to interesting applications, it leaves a number of issues unaddressed. One of the main problems is that the precise mapping between the $F$-theory data and the IIb data has never been established. For example, part of the $F$-theory data is the specification of a configuration for a three-form field $C_{3}$. Qualitatively, it has been understood that this should yield the 7-brane gauge fields and two-form tensor fields in the IIb limit, but the precise dictionary was never found. Similarly, there were problems in the comparison of tadpole constraints, instantons effects and other things.

We believe that these difficulties indicate that the usual method of analyzing the $S L(2, \mathbf{Z})$ monodromy representation really isn't the right way to think about the Sen limit. We may ask the question, what are the right tools to address this problem?

Some recent progress was obtained in [3]. Instead of focusing on the monodromy representation, the idea was to look in more detail at the degenerate $F$-theory Calabi-Yau appearing in the Sen limit. It was found for example that the IIb Calabi-Yau $X_{n-1}$, whose appearance looks somewhat mysterious in Sen's approach, emerged naturally as a certain divisor of singularities in the limit. It was also found that differential forms with logarithmic singularities play an important role in the comparison. However the picture in [3] was still too singular to establish a complete dictionary, particularly for the relation between the 7 -brane gauge fields in $\mathrm{IIb}$ and the $F$-theory three-form.

In the present paper, we continue this line of thought. As we have explained in more detail elsewhere [4], we can get a good dictionary if we construct a stable version of the degeneration. So in the present paper we will introduce a stable version of the Sen limit.

Finding a stable version turns out to be remarkably easy, as generically we only need a single blow-up of Sen's family. One finds that the elliptic Calabi-Yau splits into two pieces, a $\mathbf{P}^{1}$-bundle and a conic bundle. When applied to elliptic $K 3$-surfaces, this is the $S O(32)$ picture of $[5,6]$, as expected from the $S O(32)$ heterotic/type I/IIb orientifold duality chain 
in eight dimensions. For a more general $F$-theory compactification, the Sen limit is a generalization of the $S O(32)$ limit.

The picture that emerges is that the $D 7 / O 7$ configuration obtained by Sen is very similar to spectral data of $S O(2 n)$ type, and the dictionary between $F$-theory and the $D 7$ data of type IIb takes the form of a cylinder mapping, even when there is no $K 3$-fibration. This allows us to get a complete map between the holomorphic data in $F$-theory and type IIb. Furthermore, the picture we obtain is now completely analogous to the one for the $E_{8} \times E_{8}$ degeneration [7], and fits beautifully with the general picture for weak coupling limits advocated in [4]. As for the $E_{8} \times E_{8}$ degeneration or the general picture in [4], the $S L(2, \mathbf{Z})$ monodromy representation plays no role in the Hodge theoretic approach.

Our stable family provides a solid basis for understanding the Sen limit. In [4] we analyzed the limiting mixed Hodge structure of a certain class of degenerations, generalizing the work of [6]. The Sen limit belongs precisely to the class of degenerations considered in [4], so in Section 2.5 we specialize the analysis of [4] to this case, and recover the expected form of the IIb action.

The nilpotent orbit theorem further shows that the corrections due to smoothing the $F$-theory Calabi-Yau have the characteristic form of $D(-1)$ instanton corrections to perturbative IIb. Initial evidence for this interpretation of the corrections computed by $F$-theory was given by Sen and in [8], where the $D(-1)$-instantons were related to the instantons of SeibergWitten theory. There has been recent progress on computing these corrections directly using localization techniques in the IIb theory $[9,10]$.

\section{The IIb limit as a stable degeneration}

\subsection{Sen's description of the limit}

Let us start with some generalities. The data of an $F$-theory compactification consists of an elliptically fibered Calabi-Yau manifold $\pi: Y_{n} \rightarrow B_{n-1}$ with section. The elliptic fibration can be represented in Weierstrass form:

$$
y^{2}=x^{3}+f x+g
$$

In order to fulfill the Calabi-Yau condition, $f$ and $g$ must be sections of $K_{B}^{-4}$ and $K_{B}^{-6}$ respectively. In addition, we have to specify a suitable configuration for a three-form field $C_{3}$ with flux $G_{4}=d C_{3}$. This part of the data will be 
ignored in the next two subsections, but will play an important role later on.

In the physics literature, the Sen limit is specified as follows $[11,12]$. We parametrize the Weierstrass fibration as

$$
\begin{aligned}
& f=-\frac{1}{48}\left(\mathrm{~b}_{2}^{2}-24 \mathrm{~b}_{4}\right) \\
& g=-\frac{1}{864}\left(-\mathrm{b}_{2}^{3}+36 \mathrm{~b}_{2} \mathrm{~b}_{4}-216 \mathrm{~b}_{6}\right)
\end{aligned}
$$

for some choices of the $\mathrm{b}_{i}$, which are sections of $K_{B}^{-i}$. The coefficients are slightly different from Sen's, and were chosen so as to emphasize the relation to the $b_{i}$ appearing in Tate's algorithm. Now we introduce a parameter $t$ as follows:

$$
\begin{aligned}
& f=-\frac{1}{48}\left(\mathrm{~b}_{2}^{2}-24 t \mathrm{~b}_{4}\right) \\
& g=-\frac{1}{864}\left(-\mathrm{b}_{2}^{3}+36 t \mathrm{~b}_{2} \mathrm{~b}_{4}-216 t^{2} \mathrm{~b}_{6}\right)
\end{aligned}
$$

The discriminant is given by

$$
\begin{aligned}
\Delta & =t^{2}\left(-\mathrm{b}_{2}^{2} \mathrm{~b}_{8}-8 t \mathrm{~b}_{4}^{3}-27 t^{2} \mathrm{~b}_{6}^{2}+9 t \mathrm{~b}_{2} \mathrm{~b}_{4} \mathrm{~b}_{6}\right) \\
& \sim-\frac{1}{4} t^{2} \mathrm{~b}_{2}^{2}\left(\mathrm{~b}_{2} \mathrm{~b}_{6}-\mathrm{b}_{4}^{2}\right)+\mathcal{O}\left(t^{3}\right)
\end{aligned}
$$

Using the approximation $j(\tau) \sim \exp (-2 \pi i \tau)$ for large $\operatorname{Im}(\tau)$, we see that

$$
\exp (-2 \pi i \tau) \sim \frac{\mathrm{b}_{2}^{4}}{t^{2}\left(\mathrm{~b}_{2} \mathrm{~b}_{6}-\mathrm{b}_{4}^{2}\right)}, \quad \tau=\frac{i}{g_{s}}+a
$$

in the $t \rightarrow 0$ limit. Thus the IIb string coupling goes to zero almost everywhere, except possibly at $b_{2}=0$, and we may expect a weakly coupled IIb vacuum.

The axion is still multi-valued. In the $t \rightarrow 0$ limit all the roots of the discriminant are located at $b_{2}=0$ and $b_{2} b_{6}-b_{4}^{2}=0$. In order to relate this to IIb data, one looks at the $t \rightarrow 0$ limit of the $S L(2, \mathbf{Z})$ monodromy representation

$$
\rho: \pi_{1}(B \backslash \Delta, p t) \rightarrow S L(2, \mathbf{Z})
$$


where $p t$ is a base point. The monodromies around these roots were analyzed in $[11,12]$, with the result that

$$
\mathrm{b}_{2}=0:\left(\begin{array}{cc}
-1 & 4 \\
0 & -1
\end{array}\right), \quad \mathrm{b}_{2} \mathrm{~b}_{6}-\mathrm{b}_{4}^{2}=0:\left(\begin{array}{cc}
1 & 1 \\
0 & 1
\end{array}\right)
$$

In the type IIb theory, these $S L(2, \mathbf{Z})$ monodromies are generated by $O 7$ and $D 7$ planes respectively, so this means that we should interpret the components of the discriminant locus at $t=0$ as follows:

$$
O 7: \mathrm{b}_{2}=0, \quad D 7: \mathrm{b}_{2} \mathrm{~b}_{6}-\mathrm{b}_{4}^{2}=0
$$

Therefore we get the following picture [12]: since $b_{2}=0$ is the orientifold locus, the emerging $X_{n-1}$ is simply the double cover over $B_{n-1}$ with branch locus given by $b_{2}=0$, obtained by undoing the orientifold projection. That is, in the limit of complex structure moduli space that we discussed above, the Calabi-Yau manifold $Y_{n}$ gives rise to a Calabi-Yau $(n-1)$-fold $X_{n-1}$ given by

$$
\xi^{2}=b_{2}
$$

where $\mathrm{b}_{2} \sim K_{B_{n-1}}^{-2}, \xi \sim K_{B_{n-1}}^{-1}$. The orientifold involution is given by

$$
\xi \rightarrow-\xi
$$

and the positions of the branes on this $(n-1)$-fold are given as above. The $D 7$ locus on $X_{n-1}$ is simply the pre-image of $\mathrm{b}_{2} \mathrm{~b}_{6}-\mathrm{b}_{4}^{2}=0$ in $B_{n-1}$ under the orientifold projection.

The Sen limit has received significant attention recently, see for example [13-23].

In this approach, the appearance of $X_{n-1}$ looks somewhat mysterious, and it is not clear how physical quantities in $F$-theory are related to physical quantities on $X_{n-1}$ with 7-branes. For example in compactifications to four dimensions, we would like to know the relation between the $4 d$ superpotentials computed by $F$-theory and perturbative IIb. It is impossible to establish such relations with the methods above. We now turn to a different approach, which will allow us to derive such relations.

\subsection{Stable version of the Sen degeneration}

Let us examine the limit of the elliptic Calabi-Yau more closely. With a little bit of algebra, one finds that we can rewrite Sen's family of Weierstrass 
fibrations in the following suggestive form

$$
y^{2}=\frac{1}{1728}\left[3 \mathrm{~b}_{2}-s\right] s^{2}-\frac{\mathrm{b}_{4}}{24} t s+\frac{\mathrm{b}_{6}}{4} t^{2}
$$

Here we defined the new variable

$$
s \equiv \mathrm{b}_{2}-12 x
$$

We consider the family as an $n+1$ fold $\mathcal{Y}_{n+1}$, together with a projection $\pi_{\mathcal{Y}}: \mathcal{Y} \rightarrow \mathrm{D}$, where $\mathrm{D}$ is the disk parametrized by $t$.

As it stands, the degeneration above is too severe to extract all the relevant information. For example, we see that if we set $t=0$, then $\mathrm{b}_{4}$ and $\mathrm{b}_{6}$ drop out of the equation. As a result, information about the $D 7$-branes appears to be lost.

As we have discussed in detail in [4], we can recover this information if we instead consider a semi-stable version of the degeneration. The family above does not provide a semi-stable degeneration. The variety $\mathcal{Y}_{n+1}$ is clearly not smooth as an $(n+1)$-fold and the central fiber $Y_{0}=\pi_{\mathcal{Y}}^{-1}(0)$ has singularities worse than normal crossing. We can fix this by blowing up the family to resolve the singularities.

Our $(n+1)$-fold $\mathcal{Y}_{n+1}$ has conic singularities along the sublocus given by $y=s=t=0$, which further degenerate when $\mathrm{b}_{2}=0$. In the generic situation, we can desingularize by doing a single blow-up of $\mathcal{Y}_{n+1}$. This produces a new family $\pi_{\widetilde{\mathcal{Y}}}: \widetilde{\mathcal{Y}}_{n+1} \rightarrow \mathrm{D}$. The effect of the blow-up is to replace central fiber $Y_{0}$ of the old family $\mathcal{Y}$ by its proper transform and the exceptional divisor of the blow-up. The only remaining singularities of the central fiber are of normal crossing type, which is practically as good as a smooth variety. Thus after the blow-up, we do have a semi-stable degeneration, in fact a stable one.

Then over $t=0$, we get a new Calabi-Yau $n$-fold

$$
\widetilde{Y}_{0}=W_{T} \cup_{X_{n-1}} W_{E}
$$

where $W_{T}$ is the proper transform of the original fiber at $t=0$, and $W_{E}$ is the exceptional divisor created by the blow-up. In the generic situation, both $W_{T}$ and $W_{E}$ are smooth, and no additional blow-ups are necessary. As we will explain below, the normal crossing divisor

$$
X_{n-1}=W_{T} \cap W_{E}
$$


is a double cover of $B_{n-1}$ and should be identified with the IIb Calabi-Yau. Further, the geometry of lines on $W_{E}$ encodes the $D 7$-branes.

The original fiber at $t=0$ is given by

$$
y^{2}=\frac{1}{1728}\left[3 \mathrm{~b}_{2}-s\right] s^{2}=\frac{1}{864}\left(\mathrm{~b}_{2}+6 x\right)\left(\mathrm{b}_{2}-12 x\right)^{2}
$$

Introducing a new coordinate $\tilde{y}=y / s$, we can write this as

$$
\tilde{y}^{2}=\frac{1}{864}\left(\mathrm{~b}_{2}+6 x\right)
$$

This is the equation of a rational curve. The map $(x, \tilde{y}) \rightarrow(x, y)$ identifies the two points

$$
(x, \tilde{y})=\left(-\mathrm{b}_{2} / 12, \pm \sqrt{-\mathrm{b}_{2} / 576}\right)
$$

on each fiber. Over $b_{2}=0$, the elliptic fiber degenerates to a cusp. The proper transform $W_{T}$ replaces the double points by two distinct points, with monodromy around $b_{2}=0$. As pointed out in [3], these two points fibered over $B_{n-1}$ give precisely the Calabi-Yau $(n-1)$-fold $X_{n-1}$ which Sen identified as the IIb space-time, before orientifolding. Indeed, $X_{n-1}$ is an anti-canonical divisor of $W_{T}$, so it is automatically Calabi-Yau, and should be thought of as the 'boundary' of $W_{T}$. So it is natural to identify this with the IIb space-time. The $\mathbf{Z}_{2}$ involution used for orientifolding exchanges the two sheets and the $O 7$-planes are by definition located at the fixed points of this involution, which is given by $b_{2}=0$.

Now we discuss the geometry of the exceptional divisor $W_{E}$. It consists of a fibration of conics over $B_{n-1}$ :

$$
y^{2}=\frac{3 \mathbf{b}_{2}}{1728} u^{2}-\frac{\mathbf{b}_{4}}{24} u v+\frac{\mathbf{b}_{6}}{4} v^{2}
$$

We write this as

$$
y^{2}=\vec{u}^{T} Q \vec{u}, \quad \vec{u}=\left(\begin{array}{c}
u \\
v
\end{array}\right), \quad Q=\frac{1}{576}\left(\begin{array}{cc}
\mathrm{b}_{2} & 12 \mathrm{~b}_{4} \\
12 \mathrm{~b}_{4} & 144 \mathrm{~b}_{6}
\end{array}\right)
$$

The discriminant of this conic bundle is given by

$$
\Delta_{W_{E}}=\operatorname{det}(Q)=\frac{1}{4}\left(\mathrm{~b}_{2} \mathrm{~b}_{6}-\mathrm{b}_{4}^{2}\right)=0
$$

Over the discriminant locus, the quadratic form on the right-hand side factorizes. Thus the generic fiber of $W_{E}$ is a $\mathbf{C P}^{1}$, but over $\Delta_{W_{E}}=0$ the conic 
degenerates to a pair of lines (i.e. we get two $\mathbf{C P}^{1} \mathrm{~s}$ instead of just one). The pairs of lines intersect $X_{n-1}$ in a 'spectral divisor' $C_{n-2}$. This divisor is automatically compatible with the $\mathbf{Z}_{2}$ involution of $X_{n-1}$.

The appearance of a conic bundle is familiar from the geometric engineering of gauge groups of type $A_{n}$ or $D_{n}$. Indeed, ALE spaces of type $A_{n}$ or $D_{n}$ can both be thought of as affine conic bundles. (For exceptional gauge groups, we need elliptic fibrations). So we anticipate that $\Delta_{W_{E}}=0$ describes the $D 7$ locus, without even appealing to the (known) analysis of the limiting monodromies. We will see it more explicitly later when we use a cylinder mapping to relate modes of $C_{3}$ to a 'spectral sheaf' localized at $\Delta_{W_{E}}=0$.

(A)
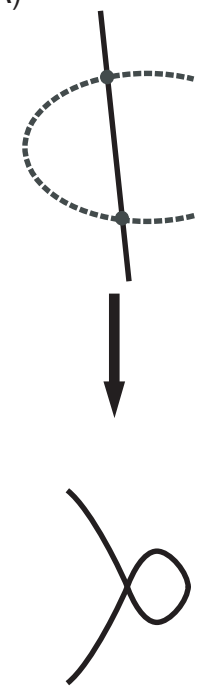

Generic
(B)
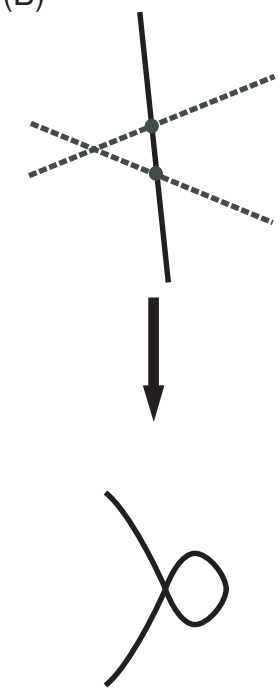

D7: $b_{2} b_{6}-b_{4}^{2}=0$
(C)
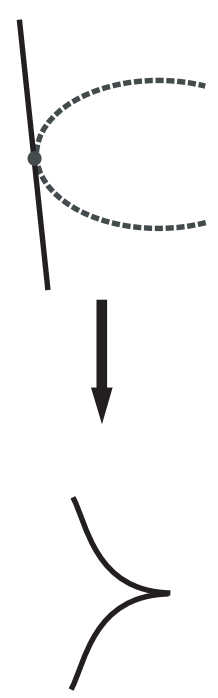

O7: $b_{2}=0$

Figure 1: Picture of elliptic fibers for $g_{s}=0$, before and after blow-up. (a) The generic fiber consists of a line and a conic. They intersect in two points, with monodromy around $b_{2}=0$. This intersection is identified with the IIb space-time. Contracting the conic leaves a nodal curve. (b) At the $D 7$ locus $b_{2} b_{6}-b_{4}^{2}=0$, the conic degenerates to a pair of lines. Blowing down the degenerate conic yields again a nodal curve. (c) At the $O 7$ locus $b_{2}=0$ the line and the conic are tangent. Upon blowing down the conic, we get a cusp. 
The situation is described in pictures in Figure 1. In the limit $t \rightarrow 0$, the generic fiber is nodal, and we get cusps over $b_{2}=0$. The blow up replaces the generic nodal fiber by two intersecting $\mathbf{P}^{1}$ 's: a line given in Equation (2.16), and a conic given in Equation (2.18). The blow-up separates the double point of the nodal curve into the two intersection points of the line with the conic. The exceptional $\mathbf{P}^{1}$ (the conic) further degenerates to a pair of lines over $b_{2} b_{6}-b_{4}^{2}=0$. And the cusp at $b_{2}=0$ is replaced by a line and a conic which are tangent, i.e the two intersection points of the line and the conic in the generic fiber coincide here.

Let us consider compactification to eight dimensions. Then $Y$ is $K 3$, $X_{1}$ is $T^{2}$ and $C_{0}$ corresponds to $2 \times 16=32$ points on the $T^{2}$, interchanged by the involution. In other words, we get an $S O(32)$ spectral cover on $X_{1}$ associated to the vector representation, and its Fourier-Mukai transform is a (highly reducible) $S O(32)$ vector bundle on $X_{1}$ of rank 32 . One may view this as a type I compactification, or as a heterotic compactification 'with vector structure,' in the language of [24]. (The $\operatorname{Spin}(32) / Z_{2}$ heterotic string on $T^{2}$ also admits compactifications without vector structure, but their type I and type IIb interpretations are a bit more subtle; see [24] and [25]).

We can get enhanced gauge symmetry by making $W_{E}$ more singular. This is particularly clear if we consider compactification to eight dimensions. To understand this, it is perhaps useful to relate our picture to some other constructions in the literature.

\subsection{Rational surfaces and $G$-bundles on an elliptic curve}

The picture we obtained is closely related to another construction in the literature. Flat $G$-bundles over an elliptic curve can be related to rational surfaces of type $G$. When $G=E_{k}$, we get the del Pezzo surface $d P_{k}$ of degree $9-k$, and the elliptic curve is an anti-canonical curve in $d P_{k}$. The generalization for $G=S O(32)$ was discussed in [6] and for general gauge groups it was discussed in [26-28].

The idea is roughly as follows. Suppose we are given a surface $S$ for which the group of line bundles $\operatorname{Pic}(S)$ is discrete, i.e. $\operatorname{Pic}(S) \cong H^{2}(S, \mathbf{Z})$, like for a rational surface. Suppose we are also given a lattice $N$, and define

$$
T=N \otimes \mathbf{C}^{*}
$$

We would like to define a $T$-bundle on $S$. To each such $T$-bundle, we can associate a line bundle (i.e. a $\mathbf{C}^{*}$-bundle), by picking a character $\chi \in \Lambda_{\text {char }}=$ $\operatorname{Hom}\left(T, \mathbf{C}^{*}\right)$. Furthermore, $\mathbf{C}^{*}$-bundles are classified by $\operatorname{Pic}(S)$, so from our 
$T$-bundle we get an element of

$$
\operatorname{Hom}\left(\Lambda_{\text {char }}, \operatorname{Pic}(S)\right)
$$

Conversely, as $T$ is abelian, such a map reconstructs a unique $T$-bundle. Our $T$-bundle splits as a sum of line bundles, and the only $T$-bundle which gets mapped to zero is the $T$-bundle whose summands are line bundles which all have their first Chern class identically zero. So $T$-bundles on $S$ are classified by $\operatorname{Hom}\left(\Lambda_{\text {char }}, \operatorname{Pic}(S)\right)$. Further note that

$$
N^{\vee}=\operatorname{Hom}(N, \mathbf{Z})=\operatorname{Hom}\left(T, \mathbf{C}^{*}\right)
$$

so we can also say that $T$-bundles on $S$ are classified by $\operatorname{Hom}\left(N^{\vee}, \operatorname{Pic}(S)\right)$.

Now suppose that $H^{2}(S, \mathbf{Z})$ has a sublattice which is isomorphic to a root lattice $\Lambda_{r t}$ for a Lie group $G$. Now we also take $N=\Lambda_{w t}$ so that $N^{\vee}=\Lambda_{\text {root }}$. Then, we get a canonical element of $\operatorname{Hom}\left(N^{\vee}, \operatorname{Pic}(S)\right)$, and therefore we get a canonical $T$-bundle on $S$. More precisely, the map is canonical up to an automorphism of $\Lambda_{\text {root }}$, which is given by the Weyl group. But $T$-bundles related by an action of the Weyl group determine the same $G$-bundle, where we identify $T$ with a maximal torus of $G$. So we get a canonical $G$-bundle on $S$.

If our rational surface $S$ contains an elliptic curve $E$, then we can restrict our canonical $G$-bundle on $S$ to get a $G$-bundle on $E$. If furthermore $[E] \in \Lambda_{r t}^{\perp} \subset \operatorname{Pic}(S)$ in the sense that $\alpha(E)=0$ for any $\alpha \in \Lambda_{r t}$, then we get a flat $G$-bundle on $E$. As shown in [26-28], all flat $G$-bundles on $E$ may be recovered in this way, and moreover there exists an essentially unique rational surface $S_{G}$ such that the moduli space of the flat $G$-bundle on $E$ equals the complex structure moduli space of $S$ keeping $E$ fixed. The surface $S_{G}$ is rational and can be constructed very explicitly. We will refer to $S_{G}$ as the rational surface of type $G$. For $G=E_{k}$, one recovers the del Pezzo surfaces.

By considering configurations of lines, we can also construct associated bundles $\mathcal{V}_{\rho}$ for each representation $\rho$ of $G$. By restriction, they yield associated bundles on $E$.

The main case of interest in this paper is type $D_{n}$, so let us spell out the relevant surfaces. We start with a Hirzebruch surface $\mathbf{F}_{1}$. We have $H^{2}\left(\mathbf{F}_{1}, \mathbf{Z}\right)=\langle b, f\rangle$ with $b^{2}=-1, f^{2}=0$, and $b \cdot f=1$. Now we blow up $n$ points $l_{1}, \ldots, l_{n}$ in general position to get $S_{D_{n}}$. The canonical bundle is given by $K=-2 b-3 f+\sum_{i} l_{i}$. The root lattice is given by

$$
\Lambda_{r t}=\{x \in \operatorname{Pic}(S) \mid x \cdot K=x \cdot f=0\}
$$


Indeed, we may take the simple roots to be given by

$$
\alpha_{1}=f-l_{1}-l_{2}, \quad \alpha_{2}=l_{1}-l_{2}, \quad \ldots, \quad \alpha_{n}=l_{n-1}-l_{n}
$$

Now we consider the elliptic curve $E$ with identity $p_{0} \in E$ representing the anti-canonical class. To this end, we first embed $E$ as an anti-canonical class in $\mathbf{P}^{2}$, using the linear system $\left|3 p_{0}\right|$. Next, we blow-up the image of $p_{0}$ in $\mathbf{P}^{2}$ to get $E$ as an anti-canonical curve in $\mathbf{F}_{1}$. Finally, we blow-up $n$ additional points $p_{i}$ on $E$, or rather their image in $\mathbf{F}_{1}$, to get $E$ as an anti-canonical curve in $S_{D_{n}}$. Recall that $-K \cdot l_{i}=1$, so the intersections by $l_{i} \cap E=p_{i}$ are the $n$ points on $E$ we introduced above.

We note some further aspects of this configuration. Each fiber $f$ intersects $E$ twice, and the sum of the two intersection points is linearly equivalent to $2 p_{0}$. So $E$ is a double covering over $b=l_{0}$. It has a natural $\mathbf{Z}_{2}$ involution interchanging the two sheets, and $p_{0}$ is one of the four fixed points. Also, the involution relates each intersection point $l_{i} \cap E=p_{i}$ to another intersection point $\left(f-l_{i}\right) \cap E=-p_{i}$. The notation $-p_{i}$ is justified as $p_{i}+\left(-p_{i}\right) \sim 2 p_{0}$, i.e. they sum to zero in the group law on $E$.

Finally, we consider the space of pairs $\left(S_{G}, E\right)$, where $S_{G}$ is a rational surface of type $G$ and $E \in|-K|$. Recall that for every $v \in \Lambda_{r t}$, we get a line bundle on $S_{G}$, which restricts to a line bundle $L_{v}$ of degree $-K \cdot v=0$ on $E$. Since the identity $p_{0} \in E$ is given, $\operatorname{Pic}^{0}(E)$ is canonically isomorphic to $E$. So we get a natural map

$$
\left(S_{G}, E\right) \rightarrow \operatorname{Hom}(\Lambda, E) / W
$$

and the image is an open dense subset. One can compactify the space of pairs $\left(S_{G}, E\right)$ by including certain singular surfaces, such that the map above extends to an isomorphism [27].

Physically it is very natural that we should compactify by including certain singular surfaces. On the heterotic side, the moduli space has boundaries where we get an enhanced gauge symmetry. On the $F$-theory side this should correspond to a singular surface, whose resolution has a chain of -2-curves orthogonal to the canonical class and intersecting in an ADE pattern. At least for the $E_{n}$ cases one can show this is exactly what happens.

Although here we only need the geometry of certain low degree curves, it is very interesting to consider curves of arbitrary degree. The correspondence predicted by heterotic/type II duality relates BPS states obtained by quantizing membranes wrapped on a curve $\Sigma$ in K3 with $\Sigma \cdot \Sigma=2 d-2$ to Dabholkar-Harvey BPS states of 'level $d$.' Their number $N(d)$ is computed 
by the left-moving partition function of a bosonic string compactified using the Narain lattice $2 \Gamma_{E_{8}} \oplus 4 H$, i.e. we have

$$
\sum_{d \geq 0} N(d) q^{d-1}=\frac{1}{\eta(q)^{24}}=q^{-1} \prod_{n>0} \frac{1}{\left(1-q^{n}\right)^{24}}
$$

We conjecture that there is an analogous correspondence for all the $S_{G}$, with the Narain lattice containing the root lattice for the group $G$.

For the case of $D_{16}=S O(32), S_{G}$ is exactly the conic bundle $W_{E}$ over $\mathbf{P}^{1}$ that we obtained in the Sen limit from a $K 3$-surface. To see this, first note that just like $S_{S O(32)}, W_{E}$ is a $\mathbf{P}^{1}$-fibration over $\mathbf{P}^{1}$ such that the fiber splits into a pair of lines $\left\{l_{i}, f-l_{i}\right\}$ for $i=1 \cdots 16$ when $\Delta_{W_{E}}=0$. In other words it is clearly a Hirzebruch surface blown up in 16 points, and the only thing left to check is the self-intersection number of the base.

It is interesting to see how the general discussion of the surfaces $S_{G}$ fits exactly with our expectations about the IIb/SO(32) limit. The intersection of $W_{E}$ with $W_{T}$ is given by the curve $v=0$ in Equation (2.18). It is a bisection of $W_{E} \rightarrow B$ with branch points over $\mathrm{b}_{2}=0$. This is precisely the elliptic curve $E$ on $S_{S O(32)}$. The rank 32 bundle associated to the vector representation of $S O(32)$ is simply given by

$$
\mathcal{V}_{S_{G}}=\bigoplus_{i} \mathcal{O}\left(l_{i}\right)_{S_{G}} \oplus \mathcal{O}\left(f-l_{i}\right)_{S_{G}}
$$

By restricting $\mathcal{V}$, and since $l_{i} \cap E=p_{i}$ and $\left(f-l_{i}\right) \cap E=2 p_{0}-p_{i}$, we get the associated $S O(32)$ bundle on $X_{1}$ given by

$$
\mathcal{V}_{E}=\bigoplus_{i} \mathcal{O}_{E}\left(p_{i}\right) \oplus \mathcal{O}_{E}\left(2 p_{0}-p_{i}\right)
$$

By tensoring with $\mathcal{O}\left(-p_{0}\right)$ we get the associated flat $S O(32)$ bundle:

$$
\mathcal{V}_{E} \otimes \mathcal{O}_{E}\left(-p_{0}\right)=\bigoplus_{i} \mathcal{O}_{E}\left(p_{i}-p_{0}\right) \oplus \mathcal{O}_{E}\left(-\left(p_{i}-p_{0}\right)\right)
$$

Note that $\left.\mathcal{O}\left(l_{0}\right)\right|_{E}=\mathcal{O}\left(p_{0}\right)$ so we could instead have started with the bundle $\mathcal{V}_{S_{G}} \otimes \mathcal{O}\left(-l_{0}\right)$ and restrict that to $E$. In any case, we see that the spectral cover (aka the D7-branes) precisely corresponds to the inverse image of $\Delta_{W_{E}}=0$ under the projection $E \rightarrow B$. One can also construct the associated spinor bundles, see [27].

We can further consider a relative version of these correspondences, by fibering over a base. This has been partially worked out for the case of 
$G=E_{k}$ discussed in $[7,29,30]$. It seems natural to expect a correspondence between the following categories:

1) pairs $\left(Z_{n-1}, V_{Z_{n-1}}\right)$, where $Z_{n-1}$ is an elliptic Calabi-Yau with section, and $V$ is a holomorphic $G$-bundles on $Z_{n-1}$, semi-stable on the generic fiber;

$2)$ triples $\left(Z_{n-1}, C_{Z_{n-1}}, L_{Z_{n-1}}\right)$ where $\left(C_{Z_{n-1}}, L_{Z_{n-1}}\right)$ is the spectral data for $V_{Z_{n-1}}$, i.e. $C_{Z_{n-1}}$ is the spectral cover and $L_{Z_{n-1}}$ is the spectral sheaf for some faithful representation of $G$.

3) triples $\left(Y_{n}, Z_{n-1},\left[C_{3}\right]\right)$ where $\left(Y_{n}, Z_{n-1}\right)$ is $\log$ Calabi-Yau and fibered by $S_{G}$, such that for each fiber we recover the dictionary between $S_{G}$ and $\left.V\right|_{E}$ discussed above. The twisting data for this fibration is the Deligne cohomology class $\left[C_{3}\right]$ which lives in a certain primitive part of the cohomology of $Y_{n}$. It corresponds to the twisting data (the spectral sheaf $L_{Z_{n-1}}$ ) of $V_{Z_{n-1}}$. Further discussion of such Deligne cohomology classes can be found in Section 2.4.

4) triples $\left(Y_{n}, Z_{n-1}, V_{Y_{n}}\right)$ where $\left(Y_{n}, Z_{n-1}\right)$ is as above, and $V_{Y_{n}}$ is a canonical $G$-bundle over $Y_{n}$ whose restriction to $Z_{n-1}$ yields $V_{Z_{n-1}}$. We expect that that the differential character $\left[\omega_{3}\left(V_{Y_{n}}\right)\right]$, where $\omega_{3}$ is the Chern-Simons three-form and $p_{1}=d \omega_{3}$ is the first Pontryagin class, is equal to the Deligne cohomology class above up to a shift by a similar class coming from the log tangent bundle.

A correspondence along these lines has been previously suggested in [31, 32]. It would be very interesting (but require some effort) to work this out more precisely.

At any rate, the Sen limit is more general than the $S O(32)$ limit, since it does not require fibrations by a $K 3$ surface. All we get in general is the structure of a conic bundle. The $A_{n}$ and $D_{n}$ surfaces are both special cases of conic bundles. So rather than investigating the above conjectural correspondences, we now move on to study conic bundles.

\subsection{The cylinder map for conic bundles}

In this section we would like to establish the IIb/F-theory duality map. Let us recall the main features of the central fiber. We have

$$
Y_{0}=W_{T} \cup_{X_{n-1}} W_{E}
$$


and $X_{n-1}=W_{T} \cap W_{E}$ is identified with the divisor of normal crossing singularities of $Y_{n}$. Furthermore, $X_{n-1} \rightarrow B_{n-1}$ is a double cover, with branch locus (i.e. $O 7$-plane locations) given by $b_{2}=0$.

For $F$-theory on a smooth Calabi-Yau $Y_{t}$, the physical data is related to the cohomology groups $H^{k}\left(Y_{t}\right)$ and their Hodge decomposition. In the limit $t \rightarrow 0$ these become the logarithmic de Rham cohomology groups $H_{\log }^{k}\left(Y_{0}\right)$, where we used the shorthand notation

$$
H_{\log }^{k}\left(Y_{0}\right)=\mathbb{H}^{k}\left(Y_{0}, \Omega_{Y_{0}}^{\bullet}\left(\log X_{n-1}\right)\right)
$$

Since $Y_{0}$ fails to be smooth and complete, these cohomology groups can be broken up into several components. This partially mirrors the fact that on the IIb side we do not have a pure Hodge structure either, but a division into closed string modes on $X_{n-1}$ and open string modes associated to the D7-branes.

More precisely, the cohomology groups $H_{\log }^{k}\left(Y_{0}\right)$ carry a natural filtration, which can be obtained as follows. On $Y_{0}$ we have the short exact sequence

$$
0 \rightarrow \Omega_{Y_{0}}^{p} \rightarrow \Omega_{Y_{0}}^{p}\left(\log \left(X_{n-1}\right)\right) \stackrel{\text { res }}{\rightarrow} \Omega_{X_{n-1}}^{p-1} \rightarrow 0
$$

where res is the Poincaré residue map. This gives rise to the long exact sequence

$$
\cdots \rightarrow H^{k-2}\left(X_{n-1}\right) \rightarrow H^{k}\left(Y_{0}\right) \rightarrow H_{\log }^{k}\left(Y_{0}\right) \rightarrow H^{k-1}\left(X_{n-1}\right) \rightarrow \cdots
$$

where the maps respect the Hodge structure, and the coboundary map $H^{k-2}\left(X_{n-1}\right) \rightarrow H^{k}\left(Y_{0}\right)$ is a Gysin map. From this we get the filtration

$$
\mathrm{W}_{k} \subseteq \mathrm{W}_{k+1}=H_{\log }^{k}\left(Y_{0}\right)
$$

where $\mathrm{W}_{k}=H^{k}\left(Y_{0}\right) / \mathrm{im}\left(H^{k-2}\left(X_{n-1}\right)\right)$, and $\mathrm{Gr}_{k+1}$ carries a pure Hodge structure. The subspace $\mathrm{W}_{k}$ does not carry a pure Hodge structure, but we can further decompose $H^{k}\left(Y_{0}\right)$ using the Mayer-Vietoris sequence

$$
\cdots \rightarrow H^{k-1}\left(X_{n-1}\right) \rightarrow H^{k}\left(Y_{0}\right) \rightarrow H^{k}\left(W_{T}\right) \oplus H^{k}\left(W_{E}\right) \stackrel{\mathrm{d}^{k}}{\rightarrow} H^{k}\left(X_{n-1}\right) \rightarrow \cdots
$$

This gives a further step in the filtration, $\mathrm{W}_{k-1} \subset \mathrm{W}_{k}$, where $\mathrm{W}_{k-1}=$ coker $\left(\mathrm{d}^{k-1}\right)$, such that $\mathrm{W}_{k-1}$ and $\mathrm{Gr}_{k}$ both carry a pure Hodge structure, and $\mathrm{Gr}_{k-1} \cong \mathrm{Gr}_{k+1}$. Together these give a two-step filtration on the cohomology 
$H_{\log }^{k}\left(Y_{0}\right)$, which is known to agree with the monodromy weight filtration on the nearby $H^{k}\left(Y_{t}\right)$. Eg. for $k=4$ we have

$$
0 \subseteq \mathrm{W}_{3} \subseteq \mathrm{W}_{4} \subseteq \mathrm{W}_{5}=H_{\log }^{4}\left(Y_{0}\right)
$$

with $\mathrm{Gr}_{3} \cong \mathrm{Gr}_{5} \cong H_{-}^{3}\left(X_{n-1}\right)$ and $\mathrm{Gr}_{4}=\operatorname{ker}\left(\mathrm{d}^{4}\right) / \operatorname{Im}\left(H^{2}\left(X_{n-1}\right)\right)$. As usual, if we are to think of $Y$ as an $F$-theory compactification rather than an $M$-theory compactification, then there are some restrictions on the allowed modes. Namely we only want to keep cohomology classes that evaluate to zero on homology classes contained in the base or that contain the elliptic fiber. We will always assume this in the following. We consider only the graded pieces in this subsection, and study the filtration in more detail in the next subsection.

We can use this decomposition of $H_{\log }^{k}\left(Y_{0}\right)$ to compare data on $Y_{0}$ to data on $X_{n-1}$. The graded pieces $\mathrm{Gr}_{k-1}$ and $\mathrm{Gr}_{k+1}$ are fairly simple to analyze. It is not hard to see that a subset of modes of $\mathrm{C}_{3}$ reproduce the IIb fields $B_{N S}^{(2)}$ and $C_{R R}^{(2)}$. Similarly one finds that a subset of the complex structure deformations of $Y_{0}$, corresponding to logarithmic $(n-1,1)$ forms with a pole along $X_{n-1}$, get mapped to involution-odd complex structure deformations of $X_{n-1}$ by taking the residue.

In this subsection we want to discuss the remaining piece of the cohomology $H_{\log }^{k}\left(Y_{0}\right)$, isomorphic to $\operatorname{Gr}_{k} \cong \operatorname{ker}\left(\mathrm{d}^{k}\right) / \operatorname{Im}\left(H^{k-2}\left(X_{n-1}\right)\right.$, which was missing in [3] as $W_{E}$ was contracted there. We want to show that there is an equivalence of the schematic form

$$
\operatorname{Gr}_{k} H_{\log }^{k}\left(Y_{0}\right) \sim H_{\mathrm{v},-}^{k-2}\left(C_{n-2}\right)
$$

at least if we restrict to modes that are allowed in $F$-theory. Here $C_{n-2}$ is the locus in $X_{n-1}$ wrapped by the $D 7$-branes. In the process one needs to deal with certain singularities of $C_{n-2}$, which we have analyzed only up to codimension two. So we will assume that $n \leq 4$.

The variety $W_{T}$ is merely a $\mathbf{P}^{1}$-fibration all of whose fibers are nonsingular. Using the Leray sequence, its cohomology is given by

$$
H^{k}\left(W_{T}\right)=H^{k}\left(B_{n-1}\right) \oplus H^{k-2}\left(B_{n-1}\right)
$$

Similarly we may compute the cohomology of $W_{E}$ using the Leray spectral sequence. The variety $W_{E}$ admits a fibration $\pi_{E}: W_{E} \rightarrow B_{n-1}$ which is a conic bundle over $B_{n-1}$, and its fibers may degenerate. Then on the $E_{2}$-page, we encounter the sheaf cohomology groups $E_{2}^{k-m, m}=H^{k-m}\left(B_{n-1}, R^{m} \pi_{E *} \mathbf{Z}\right)$, and the differential is a map $d_{2}: E_{2}^{p, q} \rightarrow E_{2}^{p+2, q-1}$. Since $R^{1} \pi_{E *} \mathbf{Z}$ vanishes, 
the sequence degenerates at $E_{2}$. We also have $R^{0} \pi_{E *} \mathbf{Z}=\mathbf{Z}_{B_{n-1}}$. Now we consider the remaining groups

$$
E_{2}^{k-2,2}=H^{k}\left(B_{n-1}, R^{2} \pi_{E *} \mathbf{Z}\right)
$$

Since taking cohomology commutes with base change in the highest degree, for any point $s$ on the base we have $\left.R^{2} \pi_{E *} \mathbf{Z}\right|_{s}=H^{2}\left(\pi_{E}^{-1}(s)\right) \cong H_{2}\left(\pi_{E}^{-1}(s)\right)$. Therefore $R^{2} \pi_{E *} \mathbf{Z} \cong \mathbf{Z} \oplus \mathcal{R}_{p}^{2}$ where the first factor is the class of the total fiber over a point $s \in B_{n-1}$, and $\mathcal{R}_{p}^{2}$ is the remainder, which is localized over the $D 7$ locus $\Delta_{W_{E}}=0$. Then we can define

$$
H_{p}^{k+2}\left(W_{E}, \mathbf{Z}\right) \equiv H^{k}\left(B_{n-1}, \mathcal{R}_{p}^{2}\right)
$$

Perhaps a simpler way to say it would be that $H_{p}^{*}\left(W_{E}\right)$ corresponds to those classes in $H^{*}\left(W_{E}\right)$ that evaluate to zero on homology classes that are contained in the base $B_{n-1}$ or that contain the fiber of $\pi_{E}: W_{E} \rightarrow B_{n-1}$. The cohomology of $W_{E}$ is thus given by

$$
H^{k}\left(W_{E}\right)=H^{k}\left(B_{n-1}\right) \oplus H^{k-2}\left(B_{n-1}\right) \oplus H_{p}^{k}\left(W_{E}\right)
$$

Now restricting to $\operatorname{ker}\left(\mathrm{d}^{k}\right)$, modding out by $\operatorname{Im}\left(H^{k-2}\left(X_{n-1}\right)\right)$, and further restricting to allowed $F$-theory modes (which are classes that evaluate to zero on homology classes contained in the base or containing the whole elliptic fiber of $\left.Y_{0}\right)$, we kill almost all the pieces of $H^{k}\left(W_{T}\right) \oplus H^{k}\left(W_{E}\right)$. We are left with the restriction of $\operatorname{ker}\left(\mathrm{d}^{k}\right)$ to $H_{p}^{k}\left(W_{E}\right)$. We would now like to give an alternative description of $H_{p}^{k}\left(W_{E}\right)$ in terms of some kind of 'spectral data.' In fact, mathematically it has been known for quite a while that the Hodge structure of a conic bundle is closely related to the Hodge structure of (a double cover of) its discriminant locus [33], and we will see that this double cover of the discriminant behaves as a brane configuration. As for del Pezzo fibrations, the isomorphism between the remaining data can be phrased as a cylinder map. For conic bundles, the cylinder map is particularly simple.

We introduce the following notation. We have the subvariety $\Delta_{W_{E}}$ in $B_{n-1}$ given by $\Delta_{W_{E}}=\mathrm{b}_{2} \mathrm{~b}_{6}-\mathrm{b}_{4}^{2}=0$, and the subvariety $R_{n-1}=\pi_{E}^{*} \Delta_{W_{E}} \subset$ $W_{E}$. We denote its normalization by $\widetilde{R}_{n-1}$; this is the analogue of the cylinder $[7,29,33,34]$. The conic degenerates to a pair of lines over $\Delta_{W_{E}}$, so $\widetilde{R}_{n-1}$ consists of pairs of (unembedded) lines fibered over $\Delta_{W_{E}}$, which further degenerate to a double line when $\mathrm{b}_{2}=\mathrm{b}_{4}=\mathrm{b}_{6}=0$. We have a natural inclusion $i: \widetilde{R}_{n-1} \rightarrow W_{E}$. We also introduce $C_{n-2}=R_{n-1} \cap X_{n-1}$. It is the pre-image of $\Delta_{W_{E}}$ in $X_{n-1}$, a double cover over $\Delta_{W_{E}}$, and should be thought 
of as the locus wrapped by the $D 7$ branes before modding out by the orientifold involution. When $n=4$ the surface $C_{n-2}$ has double point singularities along a curve $F$, which is the fixed locus of the involution. These singularities degenerate further to pinch points when $b_{2}=b_{4}=b_{6}=0$.

There is a natural map $\widetilde{R}_{n-1} \rightarrow C_{n-2}$ which replaces each line by the intersection with $X_{n-1}$. However, sometimes the two lines intersect at the same point in $X_{n-1}$, thereby yielding only a single point on $C_{n-2}$. When $n=4$ this yields exactly the curve $F$ on $C_{n-2}$. Thus the map $\widetilde{R}_{n-1} \rightarrow C_{n-2}$ factors through

$$
p_{R}: \widetilde{R}_{n-1} \rightarrow \widetilde{C}_{n-2},
$$

where $\nu: \widetilde{C}_{n-2} \rightarrow C_{n-2}$ is obtained by requiring that each line gets mapped to its own intersection point with $X_{n-1}$. It is also precisely the normalization. So two distinct lines always yield two distinct points in $\widetilde{C}_{n-2}$. Note that $\widetilde{C}_{n-2}$ itself is therefore not a subspace of $X_{n-1}$ (though it may be viewed as a subspace of the blow-up of $X_{n-1}$ along the singular locus of $C_{n-2}$ ).

There is a diffeomorphism symmetry on $\widetilde{R}_{n-1}$ which interchanges the two lines in each fiber. This descends to an involution $\rho$ on $\widetilde{C}_{n-2}$, which has fixed points only in codimension two (corresponding to a double line in $\left.\widetilde{R}_{n-1}\right)$ and $\widetilde{C}_{n-2} / Z_{2}=\Delta_{W_{E}}$. We summarize some of the relationships in the following diagram:

$$
\begin{array}{cccc}
\widetilde{R}_{n-1} & \hookrightarrow^{i} & W_{E} \\
\downarrow p_{R} & & \\
\widetilde{C}_{n-2} & & \downarrow \\
\downarrow & & \\
\Delta_{W_{E}} & \hookrightarrow & B_{n-1}
\end{array}
$$

We see that there is a natural map between $\widetilde{C}_{n-2}$ and $W_{E}$ :

$$
c \equiv i_{*} p_{R}^{*}: H_{-}^{k-2}\left(\widetilde{C}_{n-2}\right) \rightarrow H_{p}^{k}\left(W_{E}\right)
$$

Its inverse (up to a constant) is given by $c^{*}=p_{R *} i^{*}$. This map preserves the integral structure and the Hodge structure, up to a $(1,1)$ shift in the degrees. This relates the remaining data on the $F$-theory and IIb sides.

Let us see why $H_{p}^{*}\left(W_{E}\right)$ is related to the odd forms on $\widetilde{C}_{n-2}$. Given a class in $H_{p}^{*}\left(W_{E}, \mathbf{Z}\right)$, we restrict to the cylinder and then integrate over the conic fibers. By definition of $H_{p}$, the integral is equal and opposite on each 
of the two lines of the fiber, so it is odd under the exchange of the two lines. Thus the resulting class on $\widetilde{C}_{n-2}$ is odd under the orientifold involution.

Finally, in order to descend to $\mathrm{Gr}_{k}$, we have to restrict to classes in $H_{p}^{k}\left(W_{E}\right)$ that are in the kernel of $\mathrm{d}^{k}$. Let $j: C_{n-2} \rightarrow X_{n-1}$ denote the embedding. Applying the cylinder map to relate classes $H_{p}^{k}\left(W_{E}\right)$ to classes in $H_{-}^{k-2}\left(\widetilde{C}_{n-2}\right)$, the resulting classes are 'vanishing' classes in the kernel of the map $(j \circ \nu)_{*}$. We denote such classes by $H_{\mathrm{v}}^{k-2}\left(\widetilde{C}_{n-2}\right)$. Thus we find that

$$
\mathrm{Gr}_{k} H_{\log }^{k}\left(Y_{0}\right) \cap H_{p}^{k} \cong H_{\mathrm{v},-}^{k-2}\left(\widetilde{C}_{n-2}\right)
$$

as advertized. We explicitly wrote $\cap H_{p}^{k}$ in order to emphasize that in $F$ theory we have to exclude some classes which would otherwise be allowed, but in the remainder we will not always state this explicitly.

Let us discuss a bit more explicitly how this relates the bosonic fields of a $D 7$-brane wrapped on $C_{n-2}$ to the degenerate $F$-theory Calabi-Yau. As promised, this will give the geometric engineering explanation of why $\Delta_{W_{E}}=$ 0 should be identified with the $D 7$ locus of type IIb, without appealing to $S L(2, \mathbf{Z})$ monodromies (which of course gives the same answer). The bosonic fields on a D7-brane consist of a complex adjoint and a gauge field.

The complex adjoint field of the eight-dimensional gauge theory wrapped on $C_{n-2}$ describes the deformations of the $D 7$ locus. These deformations live in $H_{+}^{0}\left(\widetilde{C}_{n-2}, \nu^{*} N_{C_{n-2}}\right) \cong H_{-}^{n-2,0}\left(\widetilde{C}_{n-2}\right)$. The switch from even to odd forms is due to the fact that the isomorphism uses the holomorphic volume form on $X_{n-1}$, which is odd under the orientifold involution. By the correspondence above, this gets mapped to $H_{p}^{n-1,1}\left(W_{E}\right)$. Now $W_{E}$ carries a relative holomorphic $(n, 0)$-form $\Omega_{E}^{n, 0} \in h^{0}\left(W_{E}, K_{W_{E}}\right)$, and the deformations that keep the residue fixed are parametrized precisely by $h^{n-1,1}\left(W_{E}\right)$.

Similarly we can compare the data associated to the gauge field on the D7-brane. The Picard group of line bundles on the D7-brane is isomorphic to $H^{1}\left(\mathcal{O}_{C_{n-2}}^{*}\right)$. In the present context, we further restrict this to classes that are compatible with the orientifold involution. It is not hard to see that the Picard group sits in a short exact sequence

$$
0 \rightarrow \mathcal{J}^{1} H_{-}^{1}\left(\widetilde{C}_{n-2}\right) \rightarrow H_{-}^{1}\left(\mathcal{O}_{\widetilde{C}_{n-2}^{*}}^{*}\right) \rightarrow H_{\mathbf{Z},-}^{1,1}\left(\widetilde{C}_{n-2}\right) \rightarrow 0
$$

where

$$
\mathcal{J}^{1} H^{1}=H_{\mathbf{C}}^{1} / F^{1} H_{\mathbf{C}}^{1}+H_{\mathbf{Z}}^{1}
$$

is the Jacobian. The discrete part is given by the the first Chern class $c_{1}(L) \in$ $H_{\mathbf{Z}}^{1,1}\left(C_{n-2}\right)$, and the Jacobian parametrizes the continuous part. When the 
flux vanishes, the latter corresponds to the Wilson line moduli of the gauge field. We ignored the half-integral shift in the flux quantization law, which corresponds to an analogous shift on the $F$-theory side.

Now we apply the cylinder map. This maps our sequence (2.47) to

$$
0 \rightarrow \mathcal{J}^{2} H^{3}\left(W_{E}\right) \rightarrow \mathbb{H}^{4}\left(W_{E}, \mathcal{D}(2)\right) \rightarrow H_{\mathbf{Z}}^{2,2}\left(W_{E}\right) \rightarrow 0
$$

More precisely, we need to restrict this to $H_{p}^{*}$. The discrete part corresponds to G-flux. The continous part is given by the intermediate Jacobian

$$
\mathcal{J}^{2} H^{3}=H_{\mathbf{C}}^{3} / F^{2} H_{\mathbf{C}}^{3}+H_{\mathbf{Z}}^{3}
$$

When the G-flux vanishes, one may think of this as describing periods of the three-form $C_{3}$. Together, these two pieces of data determine a Deligne cohomology class in $\mathbb{H}^{4}\left(W_{E}, \mathcal{D}(2)\right)$, where $\mathcal{D}(2)$ is the Deligne complex $\mathcal{D}(2)=$ $\left\{(2 \pi i)^{2} \mathbf{Z} \rightarrow \Omega_{W_{E}}^{0} \rightarrow \Omega_{W_{E}}^{1}\right\}$.

The appearance of Deligne cohomology is not surprising. Just as equivalence classes of holomorphic line bundles are given by generators of the Picard group, similarly equivalence classes of the three-form field $C_{3}$ (viewed as a gerbe) are given by Deligne cohomology classes. The statement about holomorphic line bundles is a special case of the latter, as $H^{1}\left(C, \mathcal{O}^{*}\right)$ is isomorphic to the Deligne cohomology group $\mathbb{H}^{2}(C, \mathcal{D}(1))$. The $(1,1)$ shift takes this to $\mathbb{H}^{4}\left(W_{E}, \mathcal{D}(2)\right)$ on the $F$-theory side. There are various Deligne cohomology groups one could consider in connection with 2-gerbes. This particular group classifies 2-gerbes together with a kind of holomorphic connective structure. (Note that $\mathbb{H}^{2}(C, \mathcal{D}(2))$ classifies line bundles together with a holomorphic connection). Thus we see that the cylinder map solves the problem of relating equivalence classes of the 7-brane gauge fields in type IIb to equivalence classes of the three-form field in $F$-theory.

In differential geometric terms, we may think of this as follows. The map $i_{*}$ is a Gysin map, which can be represented by the Thom class $\Xi_{R}$ of $R$ in $W_{E}[35,36]$. So given a gauge field $A_{\mu} d x^{\mu}$ on $D 7$, we can promote it to a three-form of the form

$$
\mathrm{C}_{3}=\mathrm{A}_{\mu} d x^{\mu} \wedge \Xi_{R}
$$

on $W_{E}$. Similarly, given an $(n-1,0)$-form $\Phi$ on $C_{n-1}$, we can promote it to a form on $W_{E}$ which parametrizes infinitesimal complex structure deformations of $W_{E}$, keeping $X_{n-1}$ fixed:

$$
\delta \Omega_{E}=\delta \Phi \wedge \Xi_{R}
$$


Since $R$ is a divisor, $\Xi_{R}$ is of type $(1,1)$. This explains the $(1,1)$ shift in the degrees above. Conversely, the map $p_{R *}$ appearing in $c^{*}$ can be interpreted as integrating over the fibers of $p_{R}: \widetilde{R}_{n-1} \rightarrow \widetilde{C}_{n-2}$, which brings down the degree by $(1,1)$. This description of the map seems to depend on a number of choices. The formulation in terms of equivalence classes above clarifies the map between the invariant data.

\subsection{Asymptotics of the superpotential in the Sen limit}

In the previous subsections, we have described a stable version of the Sen degeneration. We saw that there was a precise dictionary between the central fiber of the stable degeneration and the perturbative IIb data on its boundary. In particular, we saw how the 7-brane gauge fields get mapped to G-flux on the central fiber.

In this subsection, we want to consider $F$-theory compactifications on Calabi-Yau four-folds and understand the limiting behaviour of the flux superpotential:

$$
W=\frac{1}{2 \pi} \int_{Y_{t}} \Omega^{4,0} \wedge \mathrm{G}
$$

We could state the problem a little more generally. Let $\Omega^{n, 0}$ denote the holomorphic volume form on $Y_{n}$. More precisely, let $\mathcal{F}^{n}$ denote the line bundle over $\mathrm{D} \backslash\{0\}$ with fiber $H^{n, 0}\left(Y_{t}\right)$ for $t \neq 0$. Let $\widetilde{\mathcal{F}}^{n}$ be its canonical extension over $t=0$, and let $\Omega^{n, 0}(t)$ be a local holomorphic frame. Then much of the interesting information about the low energy theory is contained in the periods

$$
\Pi_{I}=\int_{C_{I}} \Omega^{n, 0}
$$

So we can ask for want the behaviour of the periods in the stable degeneration limit. Depending on the value of $n$, the periods can be interpreted as computing BPS protected masses or tensions of wrapped branes. For definiteness we consider $n=4$, in which case the periods can also be interpreted as computing the value of the flux superpotential, by Poincaré duality.

The approach to the periods considered here is a generalization of [6], and a number of general aspects are explained in more detail in $[6,37]$ and [4]. For general aspects of Hodge theory see [38-40]. A nice intuitive description is given in Sections 4.3 and 4.4 of [41]. More computational 
approaches using Picard-Fuchs equations have been studied in many works, see for example [42-50].

The question of the asymptotic behaviour of the periods in a semi-stable degeneration limit is a classic problem in Hodge theory. Let us briefly review some of the relevant facts. Parallel transport of homology classes (or dually cohomology classes) around $t=0$ yields an automorphism of $H^{k}\left(Y_{t}\right)$, which can be represented as a matrix $M$. By a base change, we may assume that the monodromy is unipotent, i.e. there exists an integer $\gamma$ such that $(M-\mathbf{1})^{\gamma}=$ 0 . Let $N$ be the log of the monodromy matrix, which is then nilpotent.

In this set-up, the Schmid nilpotent orbit theorem says that the periods have the following asymptotic form in the limit $t \rightarrow 0$ :

$$
\vec{\Pi}(t) \sim e^{\frac{1}{2 \pi i} \log (t) N} \vec{\Pi}_{0}
$$

The expression on the right hand side is called the nilpotent orbit. It should be thought of as a perturbative approximation to the periods. The vector $\vec{\Pi}_{0}$ is the period map for the limiting mixed Hodge structure on $H^{4}\left(Y_{t}\right)$. Thus to find the asymptotic form of the periods, we only need a way to derive this limiting mixed Hodge structure.

By the work of Steenbrink [51], the limiting mixed Hodge structure for a semi-stable degeneration may be read off from the logarithmic cohomology groups of the central fiber. The Hodge filtration is found from the decomposition

$$
H_{\log }^{4}\left(Y_{0}\right)=\sum_{p+q=4} H^{p}\left(Y_{0}, \Omega_{Y_{0}}^{q}\left(\log X_{3}\right)\right)
$$

The monodromy weight filtration is in general a bit more complicated to describe, but for our case we already found it in Section 2.4. It is of the form

$$
0 \subseteq \operatorname{im}(N) \subseteq \operatorname{ker}(N) \subseteq H_{\log }^{4}\left(Y_{0}\right)
$$

with graded pieces $\mathrm{Gr}_{5} \cong \mathrm{Gr}_{3} \cong \operatorname{coker}\left(\mathrm{d}^{3}\right)$ and $\mathrm{Gr}_{4} \cong H_{\mathrm{v},-}^{2}\left(\widetilde{C}_{2}\right)$. From the decomposition for $H^{3}\left(W_{T}\right)$ and $H^{3}\left(W_{E}\right)$ in Section 2.4, we see that both have a piece isomorphic to $H^{3}\left(B_{3}\right) \cong H_{+}^{3}\left(X_{3}\right)$, and from the Mayer-Vietoris sequence we then find that coker $\left(\mathrm{d}^{3}\right)=H_{-}^{3}\left(X_{3}\right)$.

Now in order to find the asymptotic form of the superpotential, we write the period map for the nilpotent orbit. Let $\Omega_{0}$ be a generator for $F^{4} \cap \mathrm{W}_{5}$. In the logarithmic description, this is a logarithmic $(4,0)$ form on $Y_{0}$. The 
nilpotent orbit is given by

$$
\Omega_{0}(t) \equiv e^{\frac{1}{2 \pi i} \log (t) N} \Omega_{0}=\Omega_{0}+\frac{1}{2 \pi i} \log (t) N \Omega_{0}
$$

Here we simply expanded the exponential and used that $N^{2}=0$. Choose a basis $\left\langle e^{i}, f^{j}, g^{k}\right\rangle$ for $H^{4}\left(Y_{t}, \mathbf{Z}\right)$ which is adapted to the weight filtration. That is, $\left\langle e^{i}\right\rangle$ projects to a basis for $\mathrm{W}_{5} / \mathrm{W}_{4},\left\langle f^{j}\right\rangle$ projects to a basis for $\mathrm{W}_{4} / \mathrm{W}_{3}$, and $\left\langle g^{k}\right\rangle$ is a basis for $\mathrm{W}_{3}$. The matrix $N$ acts as $N e^{i}=g^{i}, N f^{j}=N g^{k}=0$. Fixing an isomorphism $H^{4}\left(Y_{t}, \mathbf{Z}\right) \cong \mathbf{Z}^{\operatorname{dim} H^{4}}$ using the basis above, we can write the period map as

$$
\Omega_{0}(t)=e^{i} \int_{e_{i}} \Omega_{0}+f^{j} \int_{f_{j}} \Omega_{0}+g^{k}\left(\frac{1}{2 \pi i} \log (t) \int_{e_{k}} \Omega_{0}+\int_{g_{k}} \Omega_{0}\right)
$$

We can write more explicit expressions for each of the terms using the isomorphisms of Hodge structures found in Section 2.4.

Let us consider the first term in (2.59) above. Using the isomorphism $F^{4} \mathrm{Gr}_{5} \cong F^{3} H_{-}^{3}\left(X_{3}\right)$, we can write

$$
\int_{e_{i}} \Omega_{0}=\int_{d_{i}} \Omega^{3,0}
$$

Here $\left\langle d^{i}\right\rangle$ is a basis for $H_{-}^{3}\left(X_{3}, \mathbf{Z}\right)$ which gets mapped to the image of $\left\langle e^{i}\right\rangle$ in $\mathrm{Gr}_{5}$, and $\left\langle d_{i}\right\rangle$ is its dual. The $(3,0)$ form should be thought of as the residue of the logarithmic $(4,0)$ form. Note that the holomorphic $(3,0)$ form is odd under the involution.

Now we come to the second term in (2.59). In order to understand it, let us consider the Hodge structure on $\mathrm{W}_{5} / \mathrm{W}_{3}$. We can fit it in a short exact sequence

$$
0 \rightarrow \mathrm{Gr}_{4} \rightarrow \mathrm{W}_{5} / \mathrm{W}_{3} \rightarrow \mathrm{Gr}_{5} \rightarrow 0
$$

We are interested in the $F^{4}$ part of $\mathrm{W}_{5} / \mathrm{W}_{3}$. It has a contribution from $F^{4} \mathrm{Gr}_{4}$, and another from lifting the $F^{4}$ part of $\mathrm{Gr}_{5}$ to $\mathrm{W}_{5} / \mathrm{W}_{3}$. We actually have $F^{4} \mathrm{Gr}_{4}=0$ (as well as $F^{4} \cap \mathrm{W}_{4}=0$ ), and the $F^{4}$ part of $\mathrm{Gr}_{5}$ was described in (2.60), so we only need to describe its lift to $\mathrm{W}_{5} / \mathrm{W}_{3}$. The lift is described by the extension class of (2.61). It may be written explicitly in terms of a representing homomorphism $\psi$ [52], which corresponds precisely to the second term given by $f^{j} \int_{f_{j}} \Omega_{0}$ in $(2.59)$.

In order to write a more useful expression for $\psi$, we may use the isomorphisms for the graded pieces of the Hodge structure found in Section 2.4. 
Then we see that the short exact sequence (2.61) is very similar to an extension sequence on $X_{3}$ of the form

$$
0 \rightarrow H_{\mathrm{v}}^{2}\left(C_{2}\right) \rightarrow H^{3}\left(X_{3}, C_{2}\right) \rightarrow H^{3}\left(X_{3}\right) \rightarrow 0
$$

which holds for example if $C_{2}$ is very ample, as we will assume. However, the above sequence uses $H^{2}\left(C_{2}\right)$, whereas $\mathrm{Gr}_{4}$ is related to $H^{2}\left(\widetilde{C}_{2}\right)$. They are certainly not isomorphic, since $H^{2}\left(C_{2}\right)$ doesn't even carry a pure Hodge structure. So we need the precise relation between them. ${ }^{1}$

Let us recall the precise relation between $C_{2}$ and $\widetilde{C}_{2}$. The surface $C_{2}$ is invariant under the involution, and singular along the fixed locus, which is a curve that we will call $F$. The normalization replaces this fixed locus by a double cover $\tilde{F} \rightarrow F$, which is branched at the pinch points.

By Leray, we have $H^{2}\left(\widetilde{C}_{2}, \mathbf{Z}\right)=H^{2}\left(C_{2}, \nu_{*} \mathbf{Z}\right)$. Now we can write a short exact sequence of local systems

$$
0 \rightarrow \mathbf{Z}_{C_{2}} \rightarrow \nu_{*} \mathbf{Z}_{\widetilde{C}_{2}} \rightarrow \mathbf{L}_{F} \rightarrow 0
$$

where $\mathbf{L}_{F}$ is a local system supported on $F$. This gives the long exact sequence

$$
\cdots \rightarrow H^{1}\left(\mathbf{L}_{F}\right) \rightarrow H^{2}\left(C_{2}, \mathbf{Z}\right) \rightarrow H^{2}\left(\widetilde{C}_{2}, \mathbf{Z}\right) \rightarrow H^{2}\left(\mathbf{L}_{F}\right) \rightarrow \cdots
$$

So to find the relation between $H^{2}\left(\widetilde{C}_{2}\right)$ and $H^{2}\left(C_{2}\right)$, we need to know more about $H^{2}\left(\mathbf{L}_{F}\right)$. We do this by considering the analogous short exact sequence on $F$ :

$$
0 \rightarrow \mathbf{Z}_{F} \rightarrow \nu_{*} \mathbf{Z}_{\widetilde{F}} \rightarrow \mathbf{L}_{F} \rightarrow 0
$$

In our generic situation, $\tilde{F}$ is a smooth curve with a $Z_{2}$ involution and isolated fixed points, and $F$ is its quotient. The associated long exact sequence is

$$
\cdots \rightarrow H^{2}(F, \mathbf{Z}) \rightarrow H^{2}(\tilde{F}, \mathbf{Z}) \rightarrow H^{2}\left(\mathbf{L}_{F}\right) \rightarrow 0
$$

We have that $H^{2}(F, \mathbf{Z})=H^{2}(\tilde{F}, \mathbf{Z})=\mathbf{Z}$, and the map $H^{2}(F, \mathbf{Z}) \rightarrow H^{2}(\tilde{F}, \mathbf{Z})$ is multiplication by two. Therefore we have $H^{2}\left(\mathbf{L}_{F}\right)=\mathbf{Z}_{2}$. But Hodge theory

\footnotetext{
${ }^{1}$ Another option is to work with intersection cohomology. Since the intersection cohomology of a variety is the same as that of its normalization, this will yield the same result as the approach below.
} 
depends on the rational structure, so we should kill the torsion. Going back to $(2.64)$, we see that

$$
H^{2}\left(\widetilde{C}_{2}, \mathbf{Q}\right) \cong H^{2}\left(C_{2}, \mathbf{Q}\right) / \operatorname{Im}\left(H^{1}\left(\mathbf{L}_{F}\right)\right)
$$

where we denoted the rational version of $\mathbf{L}_{F}$ by the same name. From the remaining part of the long exact sequence $(2.66)$, we see that $H^{1}\left(\mathbf{L}_{F}\right) \cong$ $H^{1}(\tilde{F}) / H^{1}(F) \cong H_{-}^{1}(\tilde{F})$.

Now when $C_{2}$ is very ample, $H^{2}\left(C_{2}\right)$ decomposes as $H_{\mathrm{v}}^{2}\left(C_{2}\right) \oplus j^{*} H^{2}\left(X_{3}\right)$, where $j: C_{2} \rightarrow X_{3}$ is the inclusion and $H_{\mathrm{v}}^{*}=\operatorname{ker}\left(j_{*}\right)$. So we can consider the image of $H_{-}^{1}(\widetilde{F})$ in $H_{\mathrm{v}}^{2}\left(C_{2}\right)$ by projecting. We again denote this by $\operatorname{Im}\left(H_{-}^{1}(\widetilde{F})\right)$. Then from $(2.62)$ we get the sequence

$$
0 \rightarrow H_{\mathrm{v}}^{2}\left(C_{2}\right) / \operatorname{Im}\left(H_{-}^{1}(\widetilde{F})\right) \rightarrow H^{3}\left(X_{3}, C_{2}\right) / \operatorname{Im}\left(H_{-}^{1}(\widetilde{F})\right) \rightarrow H^{3}\left(X_{3}\right) \rightarrow 0
$$

We can further decompose this into even and odd parts under the orientifold involution, and $\operatorname{Im}\left(H_{-}^{1}(\widetilde{F})\right)$ actually sits in the odd part, though we could have further projected if that had not been the case. Thus we get the sequence

$$
0 \rightarrow H_{\mathrm{v},-}^{2}\left(C_{2}\right) / \operatorname{Im}\left(H_{-}^{1}(\widetilde{F})\right) \rightarrow H_{-}^{3}\left(X_{3}, C_{2}\right) / \operatorname{Im}\left(H_{-}^{1}(\widetilde{F})\right) \rightarrow H_{-}^{3}\left(X_{3}\right) \rightarrow 0
$$

Now replacing $H_{\mathrm{v},-}^{2}\left(C_{2}\right) / \operatorname{Im}\left(H_{-}^{1}(\widetilde{F})\right)$ by $H_{\mathrm{v},-}^{2}\left(\widetilde{C}_{2}\right) \cong \mathrm{Gr}_{4}$, we see that $(2.69)$ above is the sequence that is equivalent to $(2.61)$.

We proceed to write the representing homomorphism $\psi$ [52] for the extension class of (2.61) or equivalently (2.69). Denote by $\left\langle c^{j}\right\rangle$ an integral basis for $H_{\mathrm{v},-}^{2}\left(C_{2}\right) / \operatorname{Im}\left(H_{-}^{1}(\widetilde{F})\right)$ which maps to the image of $\left\langle f^{j}\right\rangle$ in $\mathrm{Gr}_{4}$ under the isomorphism. The duals are cycles $c_{j} \in H_{2}\left(C_{2}\right)$ that pair to zero with $\operatorname{Im}\left(H_{-}^{1}(\widetilde{F})\right)$ and become homologically trivial when embedded in $X_{3}$. We choose a set of three-chains $\Gamma_{j}$ in $H_{3}\left(X_{3}, C_{2}\right)$ such that $\partial \Gamma_{j}=c_{j}$ and such that the image of $H_{-}^{1}(\widetilde{F})$ in $H^{3}\left(X_{3}, C_{2}\right)$ evaluates to zero on $\Gamma_{j}$. The representing homomorphism for the extension class is then given by

$$
\psi=\sum_{i} c^{j} \int_{\Gamma_{j}} \Omega^{3,0}
$$

In other words, we have found that

$$
\int_{f_{j}} \Omega_{0}=\int_{\Gamma_{j}} \Omega^{3,0}
$$


These expressions were studied in some detail in [53], under the assumption that $C_{2}$ is smooth. In that case they vary holomorphically in the moduli, and the critical locus is precisely the Noether-Lefschetz locus. In the present case it seems that these expressions still vary holomorphically, but the critical locus corresponds to the Noether-Lefschetz locus for involution odd classes on $\widetilde{C}_{2}$. As usual the chain integrals depend only on the 'endpoints' $c_{j} \subset C_{2}$, up to identifications by the periods of $\Omega^{3,0}$. Indeed if we choose any other set of $\tilde{\Gamma}_{j}$ such that $\partial \tilde{\Gamma}_{j}=c^{j}$, then $\tilde{\Gamma}_{j}-\Gamma_{j}$ is a closed cycle and the difference in the integral is a period of $\Omega^{3,0}$. So modulo the identifications, we may think of these expressions as localized on the $D 7$ locus.

An alternative approach to writing an expression for $\psi$, which is in practice probably much simpler, would be to blow up $X_{3}$ along the intersection of the $D 7$ and $O 7$ locus. The proper transform of $C_{2}$ is the normalization $\widetilde{C}_{2}$, and since $X_{3}$ is smooth the Hodge structure of $X_{3}$ lifts to the blow-up $\widetilde{X}_{3}$. However we wanted to demonstrate that it is in principle possible to work only on $X_{3}$.

Finally we consider the last term (2.59), given by $g^{k} \int_{g_{k}} \Omega_{0}$. We may think of this as being associated to the extension

$$
0 \rightarrow \mathrm{W}_{3} \rightarrow \mathrm{W}_{5} \rightarrow \mathrm{W}_{5} / \mathrm{W}_{3} \rightarrow 0
$$

We have $F^{4} \cap \mathrm{W}_{3}=0$ so the non-zero part comes entirely from the extension class. In order to write the representing homomorphism, we take an integral basis $d^{k}$ for $\mathrm{W}_{3} \cong H_{-}^{3}\left(X_{3}\right)$ and the dual basis $d_{k}$ for $H_{3,-}\left(X_{3}\right)$, and then lift the $d_{k}$ to $\mathrm{W}_{5}^{\vee}$. We can represent the lifts by four-cycles of the form $\left(c_{k, 1}, c_{k, 2}\right)$, where $c_{1}$ and $c_{2}$ are four-chains on $W_{E}$ and $W_{T}$ with $\partial c_{k, 1}=-\partial c_{k, 2}=d_{k} \in H_{3,-}\left(X_{3}\right)$. Then the representing homomorphism can be written as the integral of the logarithmic $(4,0)$ form over these fourcycles. By changing representatives, we see that up to natural ambiguities given by periods of the form $\int_{e_{i}} \Omega_{0}$ and $\int_{f_{j}} \Omega_{0}$ this integral depends only on the 'end-points' $d_{k}$. We will informally write it as

$$
\int_{g_{k}} \Omega_{0}=\int_{d_{k}} \Phi
$$

Altogether, we found that the period map for the nilpotent orbit can be written as

$$
\Omega_{0}(t)=e^{i} \int_{d_{i}} \Omega^{3,0}+f^{j} \int_{\Gamma_{j}} \Omega^{3,0}+g^{k}\left(\frac{1}{2 \pi i} \log (t) \int_{d_{k}} \Omega^{3,0}+\int_{d_{k}} \Phi\right)
$$


In order to compare with the usual expressions in perturbative type IIb, let us define

$$
\tau=\frac{1}{2 \pi i} \log (t)
$$

Then by using Poincaré duality on $X_{3}$, we see that the flux superpotential $W=\frac{1}{2 \pi} \int_{Y_{t}} \Omega^{4,0} \wedge \mathrm{G}$ has the following asymptotic form in the limit $t \rightarrow 0$ :

$$
W=\int_{X_{3}} \Omega^{3,0} \wedge \mathrm{H}+W_{D 7}+\int_{X_{3}} \Phi \wedge \widetilde{H}_{N S}+\mathcal{O}\left(\tau e^{2 \pi i \tau}\right)
$$

where we defined $\mathrm{H}=H_{R R}+\tau \widetilde{H}_{N S}$, and $W_{D 7}$ is the superpotential for $D 7$-branes wrapped on $C_{2}$ with worldvolume flux $\mathrm{F} \in H_{-}^{2}\left(C_{2}\right) / \operatorname{Im}\left(H_{-}^{1}(\widetilde{F})\right)$. More explicitly, let us write the worldvolume flux $\mathrm{F}$ of the $D 7$-brane as

$$
\mathrm{F}-\mathrm{F}_{0}=N_{j} c^{j}
$$

with $N_{j} c^{j} \in H_{\mathrm{v},-}^{2}\left(C_{2}\right) / \operatorname{Im}\left(H_{-}^{1}(\widetilde{F})\right)$ and $\mathrm{F}_{0} \in j^{*} H^{2}\left(X_{3}\right)$. Then $W_{D 7}$ is defined to be the following integral linear combination of chain integrals:

$$
W_{D 7}=\sum_{j} N_{j} \int_{\Gamma_{j}} \Omega^{3,0}
$$

An alternative way to write it is $W_{D 7}=\int_{D 7} \operatorname{Tr}\left(\phi^{2,0} \wedge\left(\bar{\partial}_{A_{0}} a+a^{2}\right)+\Phi_{0}^{2,0} a^{2}\right)$ where $a=A-A_{0}$ and $\phi=\Phi-\Phi_{0}$, by analogy with (or dimensional reduction from $)$ the expression $\omega_{C S}=\operatorname{Tr}\left(a d_{A_{0}} a+\frac{2}{3} a^{3}\right)$ for the Chern-Simons form. Again since $C_{2}$ is not smooth, this should be thought of as living on $\widetilde{C}_{2}$.

The exponential terms in (2.76) are the corrections to the nilpotent orbit. Since $\operatorname{Im}(2 \pi i \tau)$ is precisely the action of a $D(-1)$-instanton, these corrections should be interpreted as computing $D(-1)$-instanton corrections to the perturbative IIb superpotential.

\section{Further aspects of the correspondence}

In this section, we would like to explain how a few additional aspects of the relation between perturbative IIb and $F$-theory can now be given a clear explanation. 


\subsection{Tadpoles, Euler character and Chern-Schwartz-Macpherson classes}

Our approach gives a clear and conceptual way to match the Euler character of the $F$-theory Calabi-Yau with a certain tadpole constraint in type IIb. The tadpole that we want to consider is the one associated to the RR four-form field $C_{(4)}$, with flux $F_{(5)}$. In the $M$-theory description, this is the tadpole for the six-form $C_{(6)}$, with two indices along the elliptic fiber.

The tadpole gets contributions from localized D3-branes, curvature and fluxes. The constraint is given by

$$
0=d F_{(7)}=N_{D_{3}}-\frac{1}{24} \chi\left(Y_{t}\right)+\langle\mathrm{G}, \mathrm{G}\rangle
$$

where $\langle\mathrm{G}, \mathrm{G}\rangle=\frac{1}{2} \int_{Y} \mathrm{G} \wedge \mathrm{G}$. On the other hand, in perturbative IIb we have an analogous relation of the form

$$
0=d F_{(5)}=N_{D_{3}}-\frac{1}{24} \chi_{\mathrm{IIb}}(O 7, D 7)+\frac{1}{\operatorname{im}(\tau)}\langle\mathrm{H}, \mathrm{H}\rangle+\langle\mathrm{F}, \mathrm{F}\rangle
$$

Here $\chi_{\mathrm{IIb}}(O 7, D 7)$ denotes curvature contributions from the $D 7$ and $O 7$ planes, and the flux contributions are given by $\langle\mathrm{H}, \mathrm{H}\rangle=\int_{Z} \mathrm{H} \wedge \overline{\mathrm{H}}$ and $\langle\mathrm{F}, \mathrm{F}\rangle=$ $\frac{1}{2} \int_{D 7} \mathrm{~F} \wedge \mathrm{F}$. We can try to compare the individual contribution from localized branes, curvature and fluxes. This leads us to the expectation that

$$
\chi\left(Y_{t}\right) \rightarrow \chi_{\mathrm{IIb}}(O 7, D 7), \quad\langle\mathrm{G}, \mathrm{G}\rangle \rightarrow \frac{1}{\operatorname{im}(\tau)}\langle\mathrm{H}, \mathrm{H}\rangle+\langle\mathrm{F}, \mathrm{F}\rangle
$$

in the Sen limit. In this subsection, we want to explain more explicitly how the first equality comes about. The matching of the flux contribution follows from the polarization on $\mathbb{H}^{4}\left(Y_{0}, \Omega^{\bullet}\left(\log X_{3}\right)\right)$. Using the basis $\langle e, f, g\rangle$ of Section (2.5), by standard results on the monodromy weight filtration and the cylinder map we have $|(e, f, g)|^{2}=Q_{3}(e, g)+Q_{2}(f, f)$ where $Q_{3}$ is the polarization on $H^{3}\left(X_{3}, \mathbf{Z}\right)$ and $Q_{2}$ is the polarization on $H^{2}(\widetilde{C})$, so the only thing one would need to check is the normalizations.

Previously, comparisons of Euler characters were done using formulae similar to those of [54], which relate Chern classes of $Y$ to Chern classes on the base of the elliptic fibration $[13,14,55]$. The basic idea is that the Euler character of a smooth elliptic curve is zero, so the Euler character of $Y$ only gets contributions from the discriminant locus. One can then compare the resulting expression for finite $t$ with the expectation from the perturbative IIb theory. We would like to do the computation of the Euler 
character directly at $t=0$. Our computation is actually simpler, because at $t=0$ we just have $\mathbf{P}^{1}$-fibrations, whereas for $t \neq 0$ we have to deal with elliptic fibrations.

The Calabi-Yau manifold $Y_{0}=W_{T} \cup_{X_{n-1}} W_{E}$ however has normal crossing singularities, and its topology is generally different from the smooth fibers. However for a normal crossing degeneration the Betti numbers of the smooth fibers agree with the logarithmic Betti numbers of the central fiber. Thus the Euler character we want is computed on the central fiber by

$$
\sum_{k}(-1)^{k} \operatorname{dim} H_{\log }^{k}\left(Y_{0}\right)=\chi\left(W_{T}\right)+\chi\left(W_{E}\right)-2 \chi\left(X_{n-1}\right)
$$

Note that this differs from the topological Euler character of $Y_{0}$, which is given by $\chi\left(Y_{0}\right)=\chi\left(W_{T}\right)+\chi\left(W_{E}\right)-\chi\left(X_{n-1}\right)$. It gets an extra contribution $-\chi\left(X_{n-1}\right)$ from the logarithmic forms, as we can see from the exact sequence (2.34). Clearly the comparison that we want to do would not work if we used the ordinary Euler character.

There is an alternative way to derive the formula (3.4) that uses some results from the theory of Chern-Schwartz-Macpherson classes, which are defined for smooth as well as singular varieties. ${ }^{2}$ Although we strictly do not need it here, we briefly digress to explain this because it allows one to compare more general Chern classes.

The CSM classes are obtained from a natural transformation

$$
\operatorname{csm}_{*}: \mathcal{C}(V) \rightarrow A_{*}(V)
$$

on a variety $V$. Here $\mathcal{C}(V)$ is the category of constructible functions, whose elements are given by finite linear combinations $\sum m_{i} \mathbf{1}_{D_{i}}$, where $m_{i} \in \mathbf{Z}$ and $\mathbf{1}_{D_{i}}$ denotes the function which takes the value 1 on the closed subset $D_{i} \subset V$ and zero on the complement. The category $A_{*}(V)$ consists of integer linear combinations of closed subvarieties of $V$ modulo rational equivalence, i.e. linear combinations which are divisors of a rational function are set to zero. There is a further natural map from $A_{*}(V) \rightarrow H_{*}(V)$, which associates to an element of $A_{*}(V)$ its homology class.

The total CSM class is now defined as $\operatorname{csm}_{*}\left(\mathbf{1}_{V}\right)$, where $\mathbf{1}_{V}$ is the identity function on $V$. It has the following interesting normalization property: on a smooth variety, $\operatorname{csm}_{*}\left(\mathbf{1}_{V}\right)$ agrees with the (Poincaré dual of the) total Chern class of the tangent bundle. Together with naturality under pushforwards of proper maps, this determines the transformation $\operatorname{csm}_{*}$ uniquely.

\footnotetext{
${ }^{2}$ We are grateful to P. Aluffi for a very useful correspondence on CSM classes.
} 
One can show that the degree of $\operatorname{csm}_{*}\left(\mathbf{1}_{V}\right)$ always yields the topological Euler character. This is not an analytic invariant, so it tends to jump under semi-stable degeneration. Thus this is not exactly what we want.

However Verdier has shown the existence of a constructible function $\psi$, such that $\operatorname{csm}_{*}(\psi)$ is an analytic invariant. This is referred to as 'Verdier specialization' [56]. In particular, it does not jump under semi-stable degeneration. So $\operatorname{csm}_{*}(\psi)$ is the natural definition of Chern classes on our singular variety, if we want these classes to agree with classes on the smooth fibers under a degeneration. We suspect that these Chern classes can probably also be formulated in terms of logarithmic forms and the log tangent bundle $[57,58]$.

The function $\psi$ is easy to describe [59]. For smooth varieties of course we have $\psi=\mathbf{1}_{V}$. For singular varieties with normal crossing singularities, $\psi=m$ on a component of multiplicity $m$, and zero at any point which lies on multiple components.

The upshot of our discussion is that the numerical invariant of $Y_{n}$ which agrees with the Euler character of the generic fiber of our family $\mathcal{Y}$ is given by the degree of $\operatorname{csm}_{*}(\psi)$. Furthermore, we have $\psi=1$ on $W_{T} \backslash X_{n-1}$ and $W_{E} \backslash X_{n-1}$, but $\psi=0$ on $X_{n-1}$. In other words, we have

$$
\psi=\mathbf{1}_{W_{T}}+\mathbf{1}_{W_{E}}-2 \times \mathbf{1}_{X_{n-1}} .
$$

Therefore we want to calculate

$$
\operatorname{deg}\left(\operatorname{csm}_{*}(\psi)\right)=\chi\left(W_{T}\right)+\chi\left(W_{E}\right)-2 \chi\left(X_{n-1}\right)
$$

which is of course exactly the same formula we found above.

The computation is now easily done. Since $W_{T}$ is a $\mathbf{P}^{1}$-fibration without any singular fibers, we have

$$
\chi\left(W_{T}\right)=\chi\left(\mathbf{P}^{1}\right) \chi\left(B_{n-1}\right)=2 \chi\left(B_{n-1}\right) .
$$

Similarly, we can use the fact that $X_{n-1}$ is a double cover of $B_{n-1}$ branched over the orientifold locus. Assuming the orientifold locus is smooth, we clearly have

$$
\chi\left(X_{n-1}\right)=2 \chi\left(B_{n-1}\right)-\chi(O 7) .
$$

The only computation that is slightly tricky is $\chi\left(W_{E}\right)$, as the conics can degenerate. Assuming that the fiber over $\Delta_{W_{E}}$ always consists of a pair of 
lines, we would have

$$
\chi\left(W_{E}\right) \sim 2 \chi\left(B_{n-1}\right)+\chi\left(\Delta_{W_{E}}\right)
$$

since the Euler character of a smooth conic is 2, but the Euler character of a conic that has degenerated to a pair of lines is 3 . However the conic could further degenerate in higher codimension, and the type of singularity depends on the dimension $n$. To be definite, we concentrate on $F$-theory compactifications on Calabi-Yau four-folds, i.e. we take $n=4$. Then we only need to consider singularities up to codimension three.

From Equation (2.18) for $W_{E}$, we then see that when $\mathrm{b}_{2}=\mathrm{b}_{4}=\mathrm{b}_{6}=0$ the conic can further degenerate to a double line given by $y^{2}=0$, whose topological Euler character is 2 . These are exactly the ordinary double point singularities of $\Delta_{W_{E}}=0$. Let us denote the number of such points by $n_{d}$. Then we have ${ }^{3}$

$$
\chi\left(W_{E}\right)=2 \chi\left(B_{n-1}\right)+\chi\left(\Delta_{W_{E}}\right)-n_{d}
$$

Adding up the contributions, the various $\chi\left(B_{n-1}\right)$ all cancel, and we find

$$
\operatorname{deg}(\operatorname{csm}(\psi))=2 \chi(O 7)+\chi\left(\Delta_{W_{E}}\right)-n_{d}
$$

Now we can compare this with perturbative IIb. The curvature contribution to the $D 3$ tadpole which has been proposed in the IIb context is $[13,14]$ :

$$
\chi_{\mathrm{IIb}}=2 \chi(O 7)+\frac{1}{2} \chi_{0}(D 7)
$$

Here $\chi_{0}$ is defined as $\chi_{0}=\chi(\widetilde{C})-n_{d}$, where $\widetilde{C}$ is a two-fold covering of $\Delta_{W_{E}}=0$ which two-to-one generically and one-to-one at the ordinary double points. It follows that $\chi_{0}=\left(2 \chi\left(\Delta_{W_{E}}\right)-n_{d}\right)-n_{d}$. Plugging in, we see that the $F$-theory expression naturally matches with the answer expected from perturbative type IIb.

Note that the appearance of the cover $\widetilde{C} \rightarrow \Delta_{W_{E}}$ is natural from several points of view. From the $F$-theory perspective, the covering $\widetilde{C} \rightarrow \Delta_{W_{E}}$ is two-to-one precisely when the fiber of the cylinder $\widetilde{R}_{3} \rightarrow \Delta_{W_{E}}$ consists of a

\footnotetext{
${ }^{3}$ Perhaps a better way to state it is as follows: we have $\chi\left(W_{E}\right)=2 \chi\left(B_{n-1}\right)+$ $\frac{1}{2} \chi\left(\widetilde{R}_{n-1}\right)$. The fibers of $\widetilde{R}_{n-1} \rightarrow \Delta_{W_{E}}$ consists of two lines generically, and a double line over the singular locus of $\Delta_{W_{E}}=0$. Thus we have $\chi\left(W_{E}\right)=2 \chi\left(B_{n-1}\right)+$ $\chi\left(\Delta_{W_{E}}\right)-\chi_{s}\left(\Delta_{W_{E}}\right)$ where $\chi_{s}\left(\Delta_{W_{E}}\right)$ denotes the Euler character of the singular locus of $\Delta_{W_{E}}$.
} 
pair of lines, and one-to-one when the fiber of $\widetilde{R}_{3} \rightarrow \Delta_{W_{E}}$ is a double line (which is topologically a single line). On the other hand it is the natural object from the point of view of spectral covers for the vector representation of $S O(2 n)$, where it appears as the normalization of the 'naive' cover. This is why $\widetilde{C}$ already appeared in our $F$-theory/IIb duality map.

\subsection{Singularities of $S O(2 n)$ spectral covers}

We saw above that the $D 7$ locus generically has singularities in the presence of 07 -planes. This has led to a number of puzzles and claims about discrepancies, although many of these issues were resolved in [14]. In this subsection, we would like to revisit some of these issues and discuss them from the point of view of spectral covers. We would like to emphasize here that such singularities are in fact a well-known feature of almost all spectral covers, including covers for the vector representation of $S O(2 n)$, or the anti-symmetric representation of the $A_{n}$-series, and are completely natural. As such they were already encountered for example in the heterotic model building literature.

Intuitively the reason for such singularities is that over a sublocus on the base, different weights of a representation often get mapped to the same point on the corresponding spectral cover, for representation theoretic reasons. When that happens because two weights are exchanged by monodromy, we expect the cover to be smooth, but otherwise we expect singularities that can not be gotten rid off by varying parameters. It is important to remember however that spectral covers with such singularities still correspond to smooth non-abelian configurations; the singularities only appear because we insist on giving an abelianized description.

For the purpose of this paper, we are interested in the spectral cover $C$ for the $2 n$-dimensional vector representation of $S O(2 n)$, see eg. [60, 61]. It is given by the equation

$$
P_{S O(2 n)}=\operatorname{det}(\lambda I-\Phi)=\lambda^{2 n}+a_{2} \lambda^{2 n-2}+\cdots+a_{2 n}=0
$$

i.e we only have even terms and the equation is invariant under $\lambda \rightarrow-\lambda$. The $a_{i}$ are various Casimirs of the Higgs field $\Phi$. Furthermore, $a_{2 n}$ is the determinant of an anti-symmetric matrix, so it is a square, namely the square of the Pfaffian of $\Phi$. 
This cover is singular at the fixed points of the involution $\rho(\lambda)=-\lambda$. Near the singularities of (3.14) we can write the equation of $C$ as

$$
z \lambda^{2}+w^{2}=0
$$

where $z$ and $w$ are local coordinates such that $z \sim \lambda^{2 n-2}+\cdots+a_{2 n-2}$ and $a_{2 n} \sim w^{2}$. In codimension one on $C$, taking $z$ constant we see that $\lambda=w=0$ is a double point singularity. In codimension two on $C$, allowing $z$ to vary we recognize $\lambda=z=w=0$ as a cuspidal point or pinch point singularity. Again, we emphasize that these singularities of $C$ are artefacts of the abelianization. The corresponding non-abelian configurations are completely smooth (provided the Higgs bundle is stable).

The usual way to deal with the singularities of $S O(2 n)$ covers is to consider the normalization $\nu: \widetilde{C} \rightarrow C[60,61]$. In the local coordinates above, the normalization $\widetilde{C}$ is explicitly given by introducing a new coordinate $x=-w / \lambda$, and rewriting (3.15) as $z+w^{2} / \lambda^{2}=0$. In other words, we have

$$
x \lambda+w=0, \quad x^{2}+z=0
$$

Locally the surface is now well-parametrized by $x$ and $\lambda$. The map to $C$ is simply given by projecting out $x$. Under this projection the curve $\lambda=0$ on $\widetilde{C}$, which is parametrized by $x$, is mapped to $C$ by identifying $\pm x$. The projection is an isomorphism for $\lambda \neq 0$. The involution $\rho$ of $C$ lifts to the involution $\tilde{\rho}: \lambda \rightarrow-\lambda, x \rightarrow-x$ of $\widetilde{C}$. One easily checks that $\widetilde{C}$ is smooth and the fixed points of the involution are precisely the lifts of the pinch points of $C$.

To get a better sense of the spectral sheaf, let us consider the following model for an adjoint $S O(2 n)$ Higgs field:

$$
\Phi=\left(\begin{array}{cccc}
0 & a & b & 0 \\
-a & 0 & 0 & 1 \\
-b & 0 & 0 & 0 \\
0 & -1 & 0 & 0
\end{array}\right)
$$

Then $\operatorname{det}(\lambda I-\Phi)=\lambda^{4}+\left(a^{2}+b^{2}+1\right) \lambda^{2}+b^{2}$, so this works as a local model near the singularities (3.15). The spectral sheaf is given by the cokernel of $(\lambda I-\Phi)$. We find that the matrix is rank four generically, drops to rank three along generic points on the spectral cover, and drops to rank two along generic points on the double curve of the spectral cover. At the pinch point it is still rank two. So the spectral sheaf is rank one along generic points on the spectral cover $C$, and rank two along the double curve, including the pinch 
point. This means that the spectral sheaf generically lifts to a line bundle $L$ on the normalization $\widetilde{C}$, since the push-forward $\nu_{*} L$ of a line bundle is rank one generically and rank two at the image of the curve $\lambda=x^{2}+z=0$ (even at the pinch point, which is scheme theoretically a double point on this curve). The line bundle $L$ should be compatible with the orientifold involution, i.e. $\tilde{\rho}^{*} L \cong L^{\vee} \otimes K_{\tilde{C}}$.

The above behaviour of an $S O(2 n)$ spectral cover seems similar to the behaviour for the $D 7 / O 7$ planes derived by Sen. This is not surprising given the relation between $S O(32)$ type I (or heterotic) on $T^{2}$ and IIb with $D 7 / O 7$ branes on $T^{2}$, which is by $T$-duality on the $T^{2}$. By fibering the elliptic curve over a base, it is clear that the spectral data of a type I $S O(32)$ bundle must agree exactly with the $D 7 / O 7$ data of Sen.

We make a brief comment about the $D$-terms. Let $\mathcal{L}$ denote the spectral sheaf. Given what is known about principal $S O(2 n)$ Higgs bundles, we expect $D$-flatness to be equivalent to the stability condition

$$
\mathcal{K} \subset \mathcal{L} \Rightarrow \mu(\mathcal{K})<\mu(\mathcal{L})
$$

where the slope $\mu$ is defined with respect to an ample line bundle $\mathcal{O}(J)$ using the Hilbert polynomial. (We will ignore the issue of Mumford-Takemoto stability versus the slightly stronger condition of Gieseker stability here; they ar very similar but use a slightly different notion of the slope). Due to the relation $\rho^{*} \mathcal{L} \cong \mathcal{L}^{\vee}$, the slope of $\mathcal{L}$ vanishes automatically. So $\mathcal{L}$ is stable if there exist no subsheaves of positive or zero slope. Note that the subsheaf $\mathcal{K}$ is not required to be compatible with the orientifold involution. It is crucial that we use stability and not some primitiveness condition of the form $F \wedge J=0$, since $D 7 / O 7$ systems are intrinsically non-abelian at the singularities.

\subsection{D3-instantons versus $M 5$-instantons}

Another interesting issue is the comparison between $D 3$-instantons in IIb and $M 5$-instantons in $F$-theory as $t \rightarrow 0$. We will briefly review some results from $[3,62]$, and point out some issues that could not be resolved at the time. Given the explicit dictionary derived here between $F$-theory data and IIb data for $t=0$, we can now fill some of these gaps.

The $M 5$-branes in question wrap the elliptic fiber. Since the elliptic fiber splits into two in the Sen limit, the M5-brane splits into two pieces as well:

$$
M 5_{T}=M 5 \cap W_{T}, \quad M 5_{E}=M 5 \cap W_{E}
$$


Recall also that the worldvolume of the IIb D3 instanton (before orientifolding) is the intersection of the $M 5$ with the normal crossing divisor $X_{n-1}$, or equivalently it is the intersection $D 3=M 5_{T} \cap M 5_{E}$.

The contribution of an M5 instanton to the superpotential is given by the $M 5$ partition function, after factoring out four universal bosonic zero modes and two universal fermionic zero modes. The worldvolume theory of the $M 5$ consists of five scalars $\phi$, a spinor $\psi$ with eight on-shell degrees of freedom, and a chiral two-form $B^{+}$. Thus the partition function is of the schematic form

$$
Z_{M 5}=Z_{\phi} Z_{\psi} Z_{B^{+}}
$$

In the Sen limit the $M 5$ worldvolume has become reducible (with normal crossing singularities), so we have be more careful in saying what we mean by the partition function. Without a UV completion we cannot derive this from first principles, but we can give a reasonable prescription, because the singularities are rather mild. Our point of view will be that the modes of the worldvolume fields have to be glued along the intersection $M 5_{T} \cap$ $M 5_{E}$, analogous to the non-trivial gluings of reducible $D$-branes discussed in $[2,3,63]$. Then the zero modes of the various fields are described by the logarithmic cohomology groups $H_{\log }^{k}(M 5)$, in the same way as the zero modes on a smooth $M 5$-brane are described by the various $H^{k}(M 5)$.

We can again use $(2.34)$ to relate $H_{\log }^{k}(M 5)$ to the ordinary cohomology. This yields a filtration

$$
0 \subseteq \mathrm{W}_{k-1} \subseteq \mathrm{W}_{k} \subseteq \mathrm{W}_{k+1}=H_{\log }^{k}(M 5)
$$

with $\mathrm{W}_{k-1}=\operatorname{im}(N)$ and $\mathrm{W}_{k}=\operatorname{ker}(N)$, much like we saw for the $F$-theory Calabi-Yau $Y_{0}$ itself. We also have the obvious Hodge filtration on $H_{\log }^{k}(M 5)$. Together with a rational structure, these give the limiting mixed Hodge structure for the Sen limit of the M5-brane.

The D3 partition function is of the schematic form

$$
Z_{D 3}=Z_{\phi} Z_{\psi} Z_{F} Z_{\lambda_{37}}
$$

In the language of the present paper, it was shown in [3] that reduction of the $M 5$-brane along $M 5_{T}$ reproduces most of the expected degrees of freedom on a $D 3$-instanton in type IIb. More precisely, the forms used for reduction on $M 5_{T}$ in [3] can be extended along $M 5_{E}$. This is the gluing prescription 
mentioned above, and yields

$$
Z_{\phi} \rightarrow Z_{\phi}^{(5)}, \quad Z_{\psi} \rightarrow Z_{\psi}, \quad Z_{B^{+}} \rightsquigarrow Z_{\phi}^{(1)} Z_{F}
$$

It was further argued that the chiral two-form on the $M 5$-brane should have additional modes, which reproduce the partition function $Z_{\lambda_{37}}$ due to chiral currents on the intersection of the $D 3$-instanton with the $D 7$-branes. However the picture in [3] was too singular to explicitly check this. Our semistable version of the degeneration solves this problem, and as we will now see, these modes are indeed present and they simply come from reduction along the fibers of $M 5_{E}$.

To see this, let us simply restrict the cylinder map to the $D 3$-worldvolume. This yields a map

$$
c: H_{-}^{i, j}\left(\widetilde{\Sigma}_{37}\right) \rightarrow H_{p}^{i+1, j+1}\left(M 5_{E}\right)
$$

where $\Sigma_{37}=D 3 \cap D 7$, and $\nu: \widetilde{\Sigma}_{37} \rightarrow \Sigma_{37}$ is its normalization. The subscript 'p' stand for primitive, i.e. we consider the cohomology classes orthogonal to the base and the anti-canonical. The self-duality condition on the chiral two-form means that the fluxes of interest live in $H^{2,1} \oplus H^{0,3}$, and further restricting to $H_{p}$ kills any $(0,3)$ part. Under the inverse of the cylinder map, fluxes in $H^{2,1}$ get mapped to chiral currents $J=\partial \phi$ in $H^{1,0}\left(\widetilde{\Sigma}_{37}\right)$.

More precisely, what we want to do is the following. Let us fix a basis $\left\{A_{i}, B^{j}\right\}$ for $H_{3}(M 5, \mathbf{Z})$ such that $A_{i} \cap B^{j}=\delta_{i}^{j}$. We also take a basis $\omega_{i}$ for the imaginary self-dual harmonic three-forms. Then up to a suitable change of basis, we have

$$
\int_{A_{i}} \bar{\omega}_{j}=\delta_{i j}, \quad \int_{B^{j}} \bar{\omega}_{i}=\tau_{i j}
$$

where $\tau_{i j}$ is the period matrix. We also define $z^{i}$ to be periods of $C_{3}$ (asssuming that the restriction $\left.\mathrm{G}\right|_{M 5}$ is trivial in cohomology; the modification of the story when $\left.\mathrm{G}\right|_{M 5}$ is non-zero is explained below):

$$
\mathrm{C}_{3}=2 \pi z^{i} \omega_{i}+\text { c.c. }
$$

Then the partition function for $B^{+}$obtained from summing over fluxes and holomorphic factorization is essentially proportional to the theta function 
on the intermediate Jacobian of the M5-brane [64]

$$
Z_{B^{+}} \propto \Theta(\tau \mid z)=\sum \exp \left(\frac{1}{2} n^{i} n^{j} 2 \pi i \tau_{i j}+2 \pi i n^{i} z^{i}\right)
$$

So to get the asymptotic form of $Z_{B^{+}}$as $t \rightarrow 0$, we should simply substitute the periods $\tau_{i j}(t)$ and $z_{i}(t)$ for the nilpotent orbit associated to the limiting mixed Hodge structure on $H_{\log }^{3}(M 5)$. The analysis is slightly complicated but in principle it proceeds exactly like the derivation of the asymptotic form of the superpotential in Section 2.5.

Let us here simply note the main feature. From our stable version of the degeneration, we see that for $t=0$ the intermediate Jacobian admits a fibration $\mathcal{J}_{M 5} \rightarrow \mathcal{J}_{D 3}$ with fiber given by $\mathcal{J}_{\Sigma_{37}}$. Here by $\mathcal{J}_{\Sigma_{37}}$ we mean the part of the Jacobian that is odd under the orientifold involution. Then the theta function roughly speaking factorizes as $\Theta_{D 3} \Theta_{\Sigma_{37}}$; more precisely when we fix the data on the $D 3$ then we recover the theta function of $\mathcal{J}_{\Sigma_{37}}$. The theta function associated to $\mathcal{J}_{\Sigma_{37}}$ can be reinterpreted as (being proportional to) a partition function of chiral fermions. In the perturbative IIb theory, this is precisely the partition function $Z_{\lambda_{37}}$ of chiral fermions obtained from quantizing Euclidean D3-D7 strings. It is related to the chiral two-form by bosonization, i.e. we have $J=\partial \phi \sim \lambda \lambda$. Similarly, the theta function $\Theta_{D 3}$ is proportional to the partition function $Z_{F}$ of $U(1)$ Yang-Mills theory on the $D 3$ [65]. It arises essentially from the sum over worldvolume fluxes on the D3-instanton.

Thus altogether we have

$$
Z_{B^{+}} \rightarrow Z_{\phi}^{(1)} Z_{F} Z_{\lambda_{37}}
$$

and therefore, modulo subtleties in properly defining the partition functions, $Z_{M 5}$ reproduces all the pieces of $Z_{D 3}$ in the Sen limit.

As emphasized in [3], it is important to note that the intermediate Jacobian of the M5-brane is in general not isomorphic to the Jacobian of any Riemann surface. Neither does it admit a projection to a lower dimensional abelian variety, with fibers that could be interpreted as the Jacobian of a Riemann surface (or as a Prym). This happens only in special cases, analyzed here and in [3], and even then only for a piece of it as we saw above. Thus it is in general not possible, nor is it necessary, to reinterpret $Z_{B^{+}}$as a partition function of fermions, using the $2 d$ Bose-Fermi correspondence.

Now we would like to take a closer look at the vanishing behaviour. Suppose that we have zero modes for the $\lambda_{37}$ fermions in IIb. In this case the partition function vanishes, but we can still get non-zero contributions 
to derivatives of the superpotential, which inserts zero modes in the path integral. Let us see how the computations in $F$-theory and IIb are related. Again, the picture here was already argued in [3], but now we can make it more precise. It is essentially completely analogous to the relation between $F$-theory and the heterotic string (which is of course not surprising given that for K3-fibrations we recovered the $S O(32)$ degeneration).

We have

$$
Z_{\lambda_{37}}(\tau, z) \sim \int d \lambda e^{\int_{\Sigma_{37}} \lambda \bar{\partial}_{A} \lambda}
$$

Here $A$ is the restriction of the 7-brane gauge field, $z$ are its periods (assuming $\int_{\Sigma_{37}} F=0$ ), and $\tau$ describes complex structure of $\Sigma_{37}$, which is sensitive to deformations of the 7-branes or the background Calabi-Yau $X_{n-1}$.

Although the contribution to the superpotential vanishes when there are $\lambda_{37}$ zero modes (eg. when $\int_{\Sigma_{37}} F \neq 0$ ), we may still get non-trivial contributions to derivatives of the superpotential. In particular, chiral fields corresponds to infinitesimal deformations of $\tau$ and $z$, so instanton contributions to holomorphic couplings of such chiral fields involve covariant derivatives of the partition function $Z_{\lambda_{37}}(\tau, z)$ with respect to $\tau$ and $z$. Differentiating with respect to the background fields pulls down factors of $J \sim \lambda \lambda$ from the exponent in $Z_{\lambda_{37}}$. These can absorb fermion zero modes and lead to a non-vanishing path integral.

On the $M 5$-side, we may not be able to recover the desired correlators by differentiating $Z_{B^{+}}$, as the corresponding chiral fields may be massive in the $M$-theory picture. However there are still distinguished, gauge-covariant operators that we can insert in the partition function. Suppose that our M5worldvolume contains a primitive holomorphic cycle $\alpha \in H_{2}(M 5, \mathbf{Z})$. Then we can consider the Wilson surface operator ${ }^{4}$

$$
W(\alpha)=e^{\int_{\alpha} B^{+}}
$$

Note that these operators transform non-trivially under gauge transformations: Since $\delta B^{+}=\lambda_{X} \omega^{X}$, we have

$$
W(\alpha) \rightarrow e^{\int_{\alpha} B^{+}+\lambda_{X} \omega^{X}}=e^{i \lambda_{X} Q_{\alpha}^{X}} W(\alpha)
$$

\footnotetext{
${ }^{4}$ In [66] this was also phrased in terms of $M 2$ branes ending on the $M 5$-brane. We prefer to phrase it in terms of operators since we are not changing the solution of the equations of motion that we are expanding around.
} 
where $Q_{\alpha}^{X}=\int_{\alpha} \omega^{X}$. The correlation functions of such observables inserted can in principle be computed using holomorphic factorization. In fact with these insertion $W\left(\alpha_{i}\right)$, we see that they organize as a source term for $B^{+}$:

$$
\int_{M 5} B^{+} \wedge\left[\frac{\mathrm{G}}{2 \pi}+\alpha_{1}^{*}+\cdots+\alpha_{n}^{*}\right]
$$

The correlator is non-vanishing only if the expression in brackets is cohomologically trivial, i.e. if it can be expressed as $d \omega_{3}$. Integrating by parts, we get

$$
\int_{M 5} d B^{+} \wedge \omega_{3}
$$

so we can think of this as a shift of the periods $C_{3} \rightarrow C_{3}+\omega_{3}$. If $\alpha$ shrinks to zero in the $F$-theory limit, then this operator describes the coupling of the $M 5$-instanton to massless modes in $F$-theory, so it computes an M5 instanton correction to certain derivatives of the superpotential in $F$-theory. So by this mechanism, the M5 instanton may generate a contribution to a holomorphic coupling that is forbidden in perturbation theory due to gauged $U(1)$ symmetries [3].

The above prescription was motivated by comparison with the bosonized description of the heterotic string, and indeed in the degeneration limit it is easy to match this with the heterotic string or type I string (using the spectral cover description) or with type IIb. In the bosonized version, the currents $J$ on $\Sigma_{37}$ come in two types. The currents that 'live in the Cartan' are of the form $J^{a} \sim \partial \phi^{a}$. This clearly lifts to an insertion of $d B^{+}$in $Z_{B^{+}}$; it is the cylinder map we discussed earlier. But there may exist additional currents along $\Sigma_{37}$ of the form $J_{ \pm \alpha} \sim \exp ( \pm \sqrt{2} \phi \cdot \alpha)$. Due to the way that the fields vary over $\Sigma_{37}$ sometimes these currents are only defined at isolated points on $\Sigma_{37}$; this point is actually crucial for computing corrections to holomorphic couplings.

In the $M$-theory picture, these extra currents match with extra singularities of $W_{E}$ in the non-generic situation, whose blow-up yields extra cycles $\alpha \in H_{2}(M 5, \mathbf{Z}$ ) (for example when the D3-instanton intersects a stack of multiple coinciding $D 7$-branes). The heterotic/ $F$-theory duality map asserts that insertion of a current $J_{\alpha}$ lifts to the operator

$$
J_{\alpha}=e^{\sqrt{2} \phi \cdot \alpha} \rightarrow W(\alpha)=e^{\int_{\alpha} B^{+}}
$$


We see that with this dictionary, the heterotic and $M 5$-pictures match. But formulated in this way the dictionary makes sense also in the general case when there is no heterotic dual, i.e. if $W_{E}$ does not come from a $K 3$-fibration.

We would like to point out in particular that although we are still working one instanton at a time in IIb and $F$-theory, there are already interesting results about the sum over worldsheet instantons on the heterotic side. For example given a Calabi-Yau three-fold $Z$, it has been found that the sum over all instantons in any given class in $H_{2}\left(X_{3}, \mathbf{Z}\right)$ vanishes in linear sigma model constructions [67]. If we take a $\operatorname{Spin}(32) / Z_{2}$ model with vector structure and admitting an elliptic fibration, then we can straightforwardly dualize this to a IIb orientifold model using the Fourier-Mukai transform, without going through $F$-theory.

The sum over worldsheet instantons becomes a sum over 'vertical' $D 3$ instantons on the IIb side, i.e. instantons of the form $\pi_{X_{3}}^{-1}(C)$ where $\pi_{X_{3}}$ : $X_{3} \rightarrow B_{2}$ and $C$ is a curve in $B_{2}$. Horizontal $D 3$-instantons wrapping the zero section of $X_{3}$ are not included, as they map to NS5-instantons in the heterotic string. Still, the result of [67] shows that for IIb orientifold duals of linear sigma model constructions, the sum over vertical $D 3$-instantons vanishes, even though the individual contributions do not vanish. Given the state of $D 3$-instanton calculus in type IIb (or $F$-theory), this is a remarkable statement.

\section{Acknowledgements}

It is a pleasure to thank C. Doran for initial discussions, and P. Aluffi, S. Katz and J. Morgan for further discussions and correspondence related to this work. AC is supported by Simons Foundation grant no. 208258. He would like to thank the Institute of Mathematics "Simion Stoilow" of the Romanian Academy in Bucharest and the Max Planck Institute for Mathematics in Bonn for their hospitality and support during the academic year 20122013. RD acknowledges partial support by NSF grants 1304962, 0908487 and 0636606. The work of MW is supported by a Heisenberg Fellowship from the DFG.

\section{References}

[1] C. Vafa, Evidence for F theory, Nucl. Phys. B 469, 403 (1996). arXiv:hep-th/9602022. 
[2] R. Donagi and M. Wijnholt, Gluing Branes, I, arXiv:1104.2610 [hep-th].

[3] R. Donagi and M. Wijnholt, MSW Instantons, arXiv:1005.5391 [hep-th].

[4] R. Donagi, S. Katz and M. Wijnholt, Weak Coupling, Degeneration and Log Calabi-Yau Spaces, arXiv:1212.0553 [hep-th].

[5] P. S. Aspinwall and D. R. Morrison, Point - like instantons on K3 orbifolds, Nucl. Phys. B 503, 533 (1997). arXiv:hep-th/9705104.

[6] A. Clingher and J. W. Morgan, Mathematics underlying the F theory / Heterotic string duality in eight-dimensions, Commun. Math. Phys. 254, 513 (2005). arXiv:math/0308106 [math-ag].

[7] G. Curio and R. Y. Donagi, Moduli in N=1 heterotic / F theory duality, Nucl. Phys. B 518, 603 (1998). arXiv:hep-th/9801057.

[8] T. Banks, M. R. Douglas and N. Seiberg, Probing F-theory with branes, Phys. Lett. B 387, 278 (1996). arXiv: hep-th/9605199.

[9] M. Billo, L. Gallot, A. Lerda and I. Pesando, F-theoretic versus microscopic description of a conformal N=2 SYM theory, JHEP 1011, 041 (2010) arXiv:1008.5240 [hep-th].

M. Billo, M. Frau, L. Giacone and A. Lerda, Holographic nonperturbative corrections to gauge couplings, JHEP 1108, 007 (2011). arXiv:1105.1869 [hep-th].

[10] F. Fucito, J. F. Morales and D. R. Pacifici, Multi instanton tests of holography, JHEP 1109, 120 (2011). arXiv:1106.3526 [hep-th].

[11] A. Sen, F theory and orientifolds, Nucl. Phys. B 475, 562 (1996). arXiv:hep-th/9605150.

[12] A. Sen, Orientifold limit of F theory vacua, Phys. Rev. D 55, 7345 (1997). arXiv:hep-th/9702165.

[13] P. Aluffi and M. Esole, Chern class identities from tadpole matching in type IIB and F-theory, JHEP 0903, 032 (2009). arXiv:0710.2544 [hep-th].

[14] A. Collinucci, F. Denef and M. Esole, D-brane Deconstructions in IIB Orientifolds, JHEP 0902, 005 (2009). arXiv:0805.1573 [hep-th].

[15] A. Collinucci, New F-theory lifts, JHEP 0908, 076 (2009). arXiv: 0812.0175 [hep-th]. 
[16] R. Donagi and M. Wijnholt, Higgs Bundles and UV Completion in FTheory, arXiv:0904.1218 [hep-th].

[17] P. Aluffi and M. Esole, New Orientifold Weak Coupling Limits in Ftheory, JHEP 1002, 020 (2010). arXiv:0908.1572 [hep-th].

[18] M. Esole, J. Fullwood and S. -T. Yau, $D_{5}$ elliptic fibrations: nonKodaira fibers and new orientifold limits of F-theory, arXiv:1110.6177 [hep-th].

[19] M. Esole and R. Savelli, Tate Form and Weak Coupling Limits in Ftheory, JHEP (2013) 2013:27. doi:10.1007/JHEP06 (2013) 027.

[20] S. L. Cacciatori, A. Cattaneo and B. Geemen, A new CY elliptic fibration and tadpole cancellation, JHEP 1110, 031 (2011). arXiv:1107.3589 [hep-th].

[21] M. Kerstan and T. Weigand, Fluxed M5-instantons in F-theory, Nucl. Phys. B 864, 597 (2012). arXiv: 1205.4720 [hep-th].

[22] T. W. Grimm, M. Kerstan, E. Palti and T. Weigand, Massive Abelian Gauge Symmetries and Fluxes in F-theory, JHEP 1112, 004 (2011). arXiv:1107.3842 [hep-th].

[23] S. Krause, C. Mayrhofer and T. Weigand, Gauge Fluxes in F-theory and Type IIB Orientifolds, JHEP 1208, 119 (2012). arXiv:1202.3138 [hep-th].

[24] E. Witten, Toroidal compactification without vector structure, JHEP 9802, 006 (1998). arXiv:hep-th/9712028.

[25] M. Bershadsky, T. Pantev and V. Sadov, F theory with quantized fluxes, Adv. Theor. Math. Phys. 3, 727 (1999) arXiv:hep-th/9805056.

[26] Conan Leung, Naichung, ADE-bundles over rational surfaces, configuration of lines and rulings, arXiv:math/0009192.

[27] Conan Leung, Naichung; Zhang, Jiajin, Moduli of bundles over rational surfaces and elliptic curves I: simply laced cases, J. Lond. Math. Soc. (2) 80 (2009), no. 3, 750-770, arXiv:0906. 3900.

[28] Conan Leung, Naichung; Zhang, Jiajin, Moduli of bundles over rational surfaces and elliptic curves II: non-simply laced cases, Int. Math. Res. Not. IMRN 2009, no. 24, 4597-4625, arXiv:0908.1645.

[29] V. Kanev, Spectral curves, simple Lie algebras, and Prym-Tjurin varieties, Proc. Symp. Pure Math. 49 (1989), 627-645. 
[30] R. Friedman, J. W. Morgan and E. Witten, Vector bundles over elliptic fibrations, arXiv:alg-geom/9709029.

[31] R. Friedman and J. W. Morgan, Exceptional groups and del Pezzo surfaces, arXiv:math/0009155.

[32] Y. Chen and N. C. Leung, ADE bundles over surfaces with ADE singularities, arXiv:1209.4979 [math.AG].

[33] A. Beauville, Variétés de Prym et Jacobians intermédiaire, Ann. Sci. de l'Ecole Normale Sup., (4) 10 (1977), no.3, p.309 ff.

[34] R. Donagi and M. Wijnholt, Model Building with F-Theory, arXiv: 0802.2969 [hep-th].

[35] R. Bott and L. Tu, Differential forms in algebraic topology, Graduate texts in mathematics 82, Springer Verlag.

[36] P. Griffitshs and J. Harris, Principles of algebraic geometry, Wiley Interscience 1978.

[37] A. Clingher and C. F. Doran, On K3 surfaces with large complex structure, arXiv:math/0508249 [math-ag].

[38] P. A. Griffiths et. al, Topics in Transcendental Algebraic Geometry, Annals of Mathematics Studies 106, Princeton University Press, 1984.

[39] C. Voisin, Hodge theory and complex algebraic geometry I, Cambridge University Press 2002. Hodge theory and complex algebraic geometry II, Cambridge University Press 2003.

[40] C. Peters and J. Steenbrink, Mixed Hodge Structures, Springer Verlag, 1998.

[41] F. Denef, Les Houches Lectures on Constructing String Vacua, arXiv:0803.1194 [hep-th].

[42] P. Berglund and P. Mayr, Heterotic string / F theory duality from mirror symmetry, Adv. Theor. Math. Phys. 2, 1307 (1999). arXiv:hep-th/9811217.

[43] M. Alim, M. Hecht, P. Mayr and A. Mertens, Mirror Symmetry for Toric Branes on Compact Hypersurfaces, JHEP 0909, 126 (2009). arXiv:0901.2937 [hep-th].

[44] H. Jockers, P. Mayr and J. Walcher, On N=1 4d Effective Couplings for F-theory and Heterotic Vacua, Adv. Theor. Math. Phys. 14, 1433 (2010). arXiv:0912.3265 [hep-th]. 
[45] M. Alim, M. Hecht, H. Jockers, P. Mayr, A. Mertens and M. Soroush, Hints for Off-Shell Mirror Symmetry in type II/F-theory Compactifications, Nucl. Phys. B 841, 303 (2010). arXiv:0909.1842 [hep-th].

[46] H. Jockers and M. Soroush, Effective superpotentials for compact D5brane Calabi-Yau geometries, Commun. Math. Phys. 290, 249 (2009). arXiv:0808.0761 [hep-th].

[47] T. W. Grimm, T. -W. Ha, A. Klemm and D. Klevers, Computing Brane and Flux Superpotentials in F-theory Compactifications, JHEP 1004, 015 (2010). arXiv:0909.2025 [hep-th].

[48] T. W. Grimm, T. -W. Ha, A. Klemm and D. Klevers, Five-Brane Superpotentials and Heterotic / F-theory Duality, Nucl. Phys. B 838, 458 (2010). arXiv:0912.3250 [hep-th].

[49] W. Lerche and S. Stieberger, Prepotential, mirror map and $F$ theory on K3, Adv. Theor. Math. Phys. 2, 1105 (1998) [Erratum-ibid. 3, 1199 (1999)]. arXiv:hep-th/9804176.

[50] W. Lerche, On the heterotic / F theory duality in eight-dimensions, arXiv:hep-th/9910207.

[51] J. H. M. Steenbrink, Limits of Hodge structures, Invent. Math. 31, 229257 (1976).

[52] J. Carlson, Extensions of mixed Hodge structures, Journées de Géometrie Algébrique d'Angers 1979, Sijthoff and Nordhoff (1980). The geometry of the extension class of a mixed Hodge structure, Proceedings of Symposia in Pure Mathematics 46 (1987).

[53] H. Clemens, Cohomology and Obstructions II: Curves on K-trivial threefolds, arXiv:math/0206219.

[54] S. Sethi, C. Vafa and E. Witten, Constraints on low dimensional string compactifications, Nucl. Phys. B 480, 213 (1996). hep-th/9606122.

[55] B. Andreas and G. Curio, On discrete twist and four flux in $N=1$ heterotic / F theory compactifications, Adv. Theor. Math. Phys. 3, 1325 (1999). arXiv:hep-th/9908193.

[56] J. L. Verdier, Spécialisation des classes de Chern, Astérisque 82-83, 149-159 (1981). 
[57] P. Aluffi, Limits of Chow groups, and a new construction of ChernSchwartz-MacPherson classes, Pure Appl. Math. Q. 2 2006, No.4, 915941. Classes de Chern des variétés singulières, revisitées, C. R. Math. Acad. Sci. Paris 342 2006, No. 6, 405-410.

[58] X. Liao, Chern Classes Of Logarithmic Vector Fields For LocallyHomogenous Free Divisors, arXiv:1205.3843[math.AG]. Chern Classes of Logarithmic Derivations for Free Divisors with Jacobian Ideal of Linear Type, arXiv:1210.6079 [math.AG].

[59] P. Aluffi, Verdier specialization via weak factorization, Arkiv för Matematik, Springer, March 2012. arXiv: 1009.2483 [math.AG].

[60] N. Hitchin, Stable Bundles and integrable systems, Duke Math. Journal Vol. 54, no. 1, 1997.

[61] R. Donagi, Spectral Covers, arXiv:alg-geom/9505009.

[62] R. Blumenhagen, A. Collinucci and B. Jurke, On Instanton Effects in F-theory, JHEP 1008, 079 (2010). arXiv:1002.1894 [hep-th].

[63] R. Donagi and M. Wijnholt, Gluing Branes II: Flavour Physics and String Duality, arXiv:1112.4854 [hep-th].

[64] E. Witten, Five-brane effective action in $M$ theory, J. Geom. Phys. 22, 103 (1997). arXiv:hep-th/9610234.

[65] E. Witten, On $S$ duality in Abelian gauge theory, Selecta Math. 1, 383 (1995). arXiv:hep-th/9505186.

[66] J. Marsano, N. Saulina and S. Schafer-Nameki, On G-flux, M5 instantons, and $U(1) s$ in F-theory, arXiv:1107.1718 [hep-th].

[67] C. Beasley and E. Witten, Residues and world sheet instantons, JHEP 0310, 065 (2003). arXiv:hep-th/0304115. 
Department of Mathematics, University of Missouri-St. Louis ST. Louis MO 63121, USA

E-mail address: clinghera@umsl.edu

Department of Mathematics, University of Pennsylvania

Philadelphia, PA 19104-6395, USA

E-mail address: donagi@math.upenn.edu

Arnold Sommerfeld Center, Ludwig-Maximilians Universität Theresienstrasse 37, D-80333 München, Germany

E-mail address: martijn.wijnholt@physik.uni-muenchen.de 